\title{
CHAIN LEVEL FLOER THEORY AND HOFER'S GEOMETRY OF THE HAMILTONIAN DIFFEOMORPHISM GROUP*
}

\author{
YONG-GEUN OH ${ }^{\dagger}$
}

\begin{abstract}
In this paper we first apply the chain level Floer theory to the study of Hofer's geometry of the Hamiltonian diffeomorphism group in the cases without quantum contribution: we prove that any quasi-autonomous Hamiltonian path on weakly exact symplectic manifolds or any autonomous Hamiltonian path on arbitrary symplectic manifolds is length minimizing in its homotopy class with fixed ends, as long as it has a fixed maximum and a fixed minimum which are not overtwisted and has no contractible periodic orbits of period less than one. Next we give a construction of new invariant norm of the Viterbo type on the Hamiltonian diffeomorphism group of arbitrary compact symplectic manifolds.
\end{abstract}

1. Introduction. In [H1], Hofer introduced an invariant pseudo-norm on the group $\operatorname{Ham}(M, \omega)$ of compactly supported Hamiltonian diffeomorphisms of the symplectic manifold $(M, \omega)$ by putting

$$
\|\phi\|=\inf _{H \mapsto \phi}\|H\|
$$

where $H \mapsto \phi$ means that $\phi=\phi_{H}^{1}$ is the time-one map of Hamilton's equation

$$
\dot{x}=X_{H}(x),
$$

and $\|H\|$ is the function defined by

$$
\|H\|=\int_{0}^{1} \text { osc } H_{t} d t=\int_{0}^{1}\left(\max H_{t}-\min H_{t}\right) d t .
$$

He also proved that (1.1) is non-degenerate for the case $\mathbb{C}^{n}$ with respect to the standard symplectic structure. Subsequently, Polterovich [Po1] and Lalonde-McDuff [LM1] proved the non-degeneracy for the case of rational symplectic manifolds and in complete generality, respectively. We also refer to $[\mathrm{Ch}]$ for the proof in the case of tame symplectic manifolds based on the Floer homology theory of Lagrangian intersections and its simplification to [Oh4].

The invariant norm (1.1) induces a bi-invariant distance on $\mathcal{H a m}(M, \omega)$ by

$$
d(\phi, \psi):=\left\|\phi \psi^{-1}\right\|
$$

which is the Finsler distance induced by the invariant Finsler norm

$$
\|h\|=\max h-\min h
$$

on the Lie algebra $C^{\infty}(M) / \mathbb{R} \simeq T_{i d} \mathcal{H a m}(M, \omega)$ of the group $\mathcal{H a m}(M, \omega)$. A natural problem of current interest in the literature is the study of geodesics in this Finsler manifold.

Hofer [H2] proved that the path of any autonomous Hamiltonian on $\mathbb{C}^{n}$ is length minimizing as long as the corresponding Hamilton's equation has no non-constant time-one periodic orbit. This result was generalized in [MS] on general symplectic

* Received March 15, 2002; accepted for publication August 9, 2002.

${ }^{\dagger}$ Department of Mathematics, University of Wisconsin, 480 Lincoln Drive, Madison, WI 53706, USA (oh@math.wisc.edu). Partially supported by the NSF Grant \# DMS-9971446 and by a grant of the Korean Young Scientist Prize. 
manifolds for the case of slow autonomous Hamiltonians among the paths homotopic with fixed ends: According to [En], [MS] and [Mc], an autonomous Hamiltonian is called slow if it has no non-constant contractible periodic orbit of period less than 1 and the linearized flow at each fixed point is not over-twisted i.e., has no closed trajectory of period less than one

We call two Hamiltonians $G$ and $F$ are called equivalent if there exists a family $\left\{F^{s}\right\}_{0 \leq s \leq 1}$ such that

$$
\phi_{F^{s}}^{1}=\phi_{G}^{1}
$$

for all $s \in[0,1]$. We denote $G \sim F$ in that case and say that two Hamiltonian paths $\phi_{G}^{t}$ and $\phi_{F}^{t}$ are homotopic to each other with fixed ends, or just homotopic to each other when there is no danger of confusion.

In the present paper, we study the length minimizing property of quasi-autonomous Hamiltonian paths: Such a Hamiltonian path was proven to be a geodesic in the sense of the Finsler geometry [LM2] (up to the time reparameterization). We refer to [Po2] for the precise variational definition of geodesics from the first principle and an elegant proof of this latter fact. We will just borrow the theorems from [LM2] or [Po2] for a concrete description of geodesics in terms of the quasi-autonomous Hamiltonian.

Definition 1.1. A Hamiltonian $H$ is called quasi-autonomous if there exists two points $x^{-}, x^{+} \in M$ such that

$$
H\left(x^{-}, t\right)=\min _{x} H(x, t), \quad H\left(x^{+}, t\right)=\max _{x} H(x, t)
$$

for all $t \in[0,1]$.

It has been proven in [BP], [LM2], [Po2] that a path $\left\{\phi^{t}\right\}$ is a geodesic in the variational sense iff the corresponding Hamiltonian $H$ is locally quasi-autonomous. Based on this theorem, we just say that a geodesic is the Hamiltonian path generated by a locally quasi-autonomous Hamiltonian.

We now recall Lalonde-McDuff's necessary condition on the stability of geodesics. In [Corollary 4.11, LM2] salonde-McDuff proved that for a generic $\phi$ in the sense that all its fixed points are isolated, any stable geodesic $\phi_{t}, 0 \leq t \leq 1$ from the identity to $\phi$ must have at least two fixed points at which the linearized isotopy has no non-constant closed trajectory in time less than 1 in the sense of Definition 1.2 below.

Definition 1.2. Let $H: M \times[0,1] \rightarrow \mathbb{R}$ be a Hamiltonian which is not necessarily time-periodic and $\phi_{H}^{t}$ be its Hamiltonian flow.

(1) We call a point $p \in M$ a time $T$ periodic point if $\phi_{H}^{T}(p)=p$. We call $t \in[0, T] \mapsto \phi_{H}^{t}(p)$ a contractible time $T$-periodic orbit if it is contractible.

(2) When $H$ has a fixed critical point $p$ over $t \in[0, T]$, we call $p$ over-twisted as a time $T$-periodic orbit if its linearized flow $d \phi_{H}^{t}(p) ; t \in[0, T]$ on $T_{p} M$ has a closed trajectory of period less than $T$.

The following is the main result of the present paper.

Theorem I. Suppose that $G$ is a quasi-autonomous Hamiltonian such that

(i) it has no nonconstant contractible periodic orbits of period less than one,

(ii) it has a fixed minimum and a fixed maximum which are not over-twisted.

Then its Hamiltonian path $\phi_{G}^{t}$ is length minimizing in its homotopy class with fixed ends for $0 \leq t \leq 1$, in cases

(1) $(M, \omega)$ is weakly exact, i.e., $\left.\omega\right|_{\pi_{2}(M)}=0$ or 
(2) $G$ is autonomous.

The case (1) extends the result by Siburg [Si] on $\mathbb{R}^{2 n}$, and (2) extends Entov's [En] and Lalonde-McDuff-Slimowitz's result [MS] for the slow autonomous case in that it removes the slowness assumption in the case of autonomous Hamiltonian. In fact the proof of Theorem I (more specifically, Step II in $\S 7$ ) shows that the length minimizing property is stable under the $C^{2}$-small perturbation of Hamiltonians of the type in the theorem and so the theorem still holds for $C^{2}$-small perturbations of the autonomous Hamiltonian $G$. When there exists quantum contribution, whether Theorem I holds is still to be seen.

Our proof of Theorem I will be based on the Floer homology theory which has been established on general symplectic manifolds by now [FOn], [LT], [Ru]. The idea of studying the length minimizing property using the Floer theory was introduced by Polterovich [Po2] for the case of small autonomous Hamiltonians when the action functional is single valued as in the case of exact symplectic manifolds. We generalize his scheme to the case of quasi-autonomous Hamiltonian paths when the action functional is not single valued.

We first summarize Polterovich's scheme of the proof for the case of small autonomous Hamiltonian when the symplectic form $\omega$ is exact, say $\omega=-d \theta$. A crucial idea behind his scheme is to relate the norm $\|h\|=h\left(x^{+}\right)-h\left(x^{-}\right)$with two homologically essential critical values of the action functional

$$
\mathcal{A}_{h}(\gamma)=\int_{\gamma} \theta-\int_{0}^{1} h(\gamma(t)) d t
$$

corresponding to the maximum and minimum points $x^{+}$and $x^{-}$of the function $h$, which is precisely $-h\left(x^{-}\right)$and $-h\left(x^{+}\right)$respectively. This is carried out first by proving some existence result for the Floer continuity equation

$$
\left\{\begin{array}{l}
\frac{\partial u}{\partial \tau}+J\left(\frac{\partial u}{\partial t}-X_{L^{\rho(\tau)}}(u)\right)=0 \\
u(-\infty) \in \text { Crit } k, u(\infty)=x^{+}
\end{array}\right.
$$

where $L^{s}$ is the linear homotopy

$$
L^{s}=(1-s) k+s F, s \in[0,1]
$$

for the small autonomous Hamiltonian $k$ and the arbitrary Hamiltonian $F$ with $F \sim h$, and then by making some calculations involving the action functional and the solution of (1.4). (Similar calculations of this sort were previously employed by Chekanov [Ch] and by the present author $[\mathrm{Oh} 3,5]$.) For the existence result, Polterovich exploits the fact that when $h$ is sufficiently small, then the Floer complex is diffeomorphic to the Morse complex of $h$ and so the maximum point on the compact manifold $M$ is homologically essential, which in turn is translated into the existence of a solution of (1.4), via the fact that the Floer complexes of $h$ and $F$ are conjugate to each other (see Proposition 5.3), when $F \sim h$.

When we try to use the Floer homology theory in the study of quasi-autonomous Hamiltonian paths, the first obvious point we need to take care of is that the Hamiltonian may not be one-periodic. This can be taken care of using a canonical modification of Hamiltonians into the time periodic ones without changing their time-one maps and the quasi-autonomous property (see Lemma 5.2 for the precise statements). 
There are many difficulties to overcome for the non-autonomous Hamiltonians especially when the action functional is not single-valued. However using the full power of the Floer homology theory developed by now (in the level of chain, though) and an idea of the mini-max theory via the Floer homology developed by the author in [Oh3,5], we again reduce the proof of Theorem I to a similar existence result (Proposition 5.3) for (1.4) where $h$ is replaced by a quasi-autonomous Hamiltonian. Unlike the small autonomous case, such an existence result is highly non-trivial (even in the autonomous case) for large Hamiltonians. In fact, the method we employ to prove the existence theorem heavily relies on the extensive chain level Floer theory. The latter turns out to carry applicability much wider than as we use in the present paper and leads us to the construction of the spectral invariants on arbitrary compact symplectic manifolds (See $\S 8$ and $[\mathrm{Oh} 7]$ ).

The proof of Theorem I will then be carried out by a continuation argument over the homotopy

$$
\epsilon k \mapsto \epsilon_{0} G^{\epsilon_{0}} \mapsto G \mapsto F
$$

combined with a delicate mini-max argument via the Floer homology over the adiabatic homotopy. One important point that we are exploiting in the first step is that when the Hamiltonian is $C^{2}$-small as in the case of $\epsilon G^{\epsilon}$ for $\epsilon$ sufficiently small, the Floer boundary operator is decomposed into

$$
\partial=\partial_{0}+\partial^{\prime}
$$

where $\partial_{0}$ is the classical contribution and $\partial^{\prime}$ is the quantum contribution (see $\S 5$, and [Oh2] in the context of Lagrangian intersections ). This enables us to define the concept of the local Floer homology which is invariant under the local continuation (see [Oh1] in the context of Lagrangian submanifolds). In general $\partial^{\prime}$ is not zero, but is so either when $(M, \omega)$ is weakly exact, or when the Hamiltonian is $C^{2}$-small and autonomous which is due to the extra $S^{1}$ symmetry (see [Fl2], [FHS], [FOn], [LT]). This is one place where we used the hypotheses in Theorem I.

The second ingredient we use in this paper is several versions of the Non pushing down lemma culminating in Proposition 7.14. In fact this kind of non-pushing down lemma is the heart of the matter in the chain level Floer theory (see [Oh7] for more such arguments in general). The proofs of these Non-pushing down lemmas use the above hypothesis in a more serious way and also use the concept of adiabatic homotopy and adiabadic chain map. The third ingredient is a Floer theoretic version of the Handle sliding lemma (Proposition 6.3). These tools enable us to develop a mini-max theory of the action functional in the non-exact case. In the much simpler setting of the (weakly-)exact case where the action functional is single valued, similar mini-max idea was previously developed by the author in [Oh3,5] for the Lagrangian submanifolds on the cotangent bundle, and subsequently by Schwarz [Sc] for the Hamiltonian diffeomorphisms on symplectically aspherical symplectic manifolds. As an application of this mini-max theory, we prove the following construction of the spectral invariants

TheOREM II. For each cohomology class $0 \neq a \in H^{*}(M, \mathbb{Q})$ and Hamiltonian $H$, there exists an invariant $\rho(H ; a)$ such that $\rho(H ; a) \in S p e c H$, and the assignment $H \mapsto \rho(H ; a)$ is $C^{0}$-continuous.

In a sequel [Oh7] to the present paper, we have further developed the techniques used here and applied them to extend the definition of these spectral invariants to the arbitrary quantum cohomology classes $a \in Q H^{*}(M)$. These are then applied to 
construct an invariant norm and to obtain a new lower bound for the Hofer norm and to the study of the length minimizing property of Hofer's geodesics.

We would like to thank L. Polterovich for introducing us to the idea of studying length minimizing property of geodesics in terms of the Floer theory during his visit of KIAS Seoul, Korea, in April 2000 and giving us a copy of his book [Po2] before its publication. We also thank D. McDuff for sending us the preprints $[\mathrm{MS}],[\mathrm{Mc}]$ and informing us that the proof in [LM2] already proves the local length minimizing property of geodesics once construction of Gromov-Witten invariants on general symplectic manifolds is established. We would also like to thank her for several helpful e-mail communications. Finally we like to thank the referee for several suggestions to improve the presentation of the paper.

2. Normalization of the Hamiltonian and the action spectrum. Let $\Omega_{0}(M)$ be the set of contractible loops and $\widetilde{\Omega}_{0}(M)$ be its standard covering space in the Floer theory. We recall the definition of this covering space from [HS] here. Note that the universal covering space of $\Omega_{0}(M)$ can be described as the set of equivalence classes of the pair $(\gamma, w)$ where $\gamma \in \Omega_{0}(M)$ and $w$ is a map from the unit disc $D=D^{2}$ to $M$ such that $\left.w\right|_{\partial D}=\gamma$ : the equivalence relation to be used is that $\left[\bar{w} \# w^{\prime}\right]$ is zero in $\pi_{2}(M)$.

Following Seidel [Se], we say that $(\gamma, w)$ is $\Gamma$-equivalent to $\left(\gamma, w^{\prime}\right)$ iff

$$
\omega\left(\left[w^{\prime} \# \bar{w}\right]\right)=0 \quad \text { and } c_{1}([w \# \bar{w}])=0
$$

where $\bar{w}$ is the map with opposite orientation on the domain and $w^{\prime} \# \bar{w}$ is the obvious glued sphere. And $c_{1}$ denotes the first Chern class of $(M, \omega)$. We denote by $[\gamma, w]$ the $\Gamma$-equivalence class of $(\gamma, w)$ and by $\pi: \widetilde{\Omega}_{0}(M) \rightarrow \Omega_{0}(M)$ the canonical projection. We also call $\widetilde{\Omega}_{0}(M)$ the $\Gamma$-covering space of $\Omega_{0}(M)$. The action functional $\mathcal{A}_{0}: \widetilde{\Omega}_{0}(M) \rightarrow$ $\mathbb{R}$ is defined by

$$
\mathcal{A}_{0}([\gamma, w])=-\int w^{*} \omega
$$

Two $\Gamma$-equivalent pairs $(\gamma, w)$ and $\left(\gamma, w^{\prime}\right)$ have the same action and so the action is well-defined on $\widetilde{\Omega}_{0}(M)$. When a periodic Hamiltonian $H: M \times(\mathbb{R} / \mathbb{Z}) \rightarrow \mathbb{R}$ is given, we consider the functional $\mathcal{A}_{H}: \widetilde{\Omega}(M) \rightarrow \mathbb{R}$ by

$$
\mathcal{A}_{H}([\gamma, w])=\mathcal{A}_{0}(\gamma, w)-\int H(\gamma(t), t) d t
$$

Here the sign convention is chosen to be consistent with that of $[\mathrm{Oh} 3,5]$,

$$
\mathcal{A}_{H}(\gamma)=\int_{\gamma} \theta-\int_{0}^{1} H(\gamma(t), t) d t
$$

where $\omega=-d \theta$ for the canonical one form $\theta=p d q$ on the cotangent bundle which in turn is precisely the classical mechanics Lagrangian on the cotangent bundle.

We would like to note that under this convention the maximum and minimum are reversed when we compare the action functional $\mathcal{A}_{G}$ and the (quasi-autonomous) Hamiltonian $G$.

We denote by $\operatorname{Per}(H)$ the set of periodic orbits of $X_{H}$. 
Definition 2.1 [Action Spectrum]. We define the action spectrum of $H$, denoted as $\operatorname{Spec}(H) \subset \mathbb{R}$, by

$$
\operatorname{Spec}(H):=\left\{\mathcal{A}_{H}(z, w) \in \mathbb{R} \mid[z, w] \in \widetilde{\Omega}_{0}(M), z \in \operatorname{Per}(H)\right\},
$$

i.e., the set of critical values of $\mathcal{A}_{H}: \widetilde{\Omega}(M) \rightarrow \mathbb{R}$. For each given $z \in \operatorname{Per}(H)$, we denote

$$
\operatorname{Spec}(H ; z)=\left\{\mathcal{A}_{H}(z, w) \in \mathbb{R} \mid(z, w) \in \pi^{-1}(z)\right\} .
$$

Note that $\operatorname{Spec}(H ; z)$ is a principal homogeneous space modeled by the period group of $(M, \omega)$

$$
\Gamma_{\omega}=\Gamma(M, \omega):=\left\{\omega(A) \mid A \in \pi_{2}(M)\right\}
$$

and

$$
\operatorname{Spec}(H)=\cup_{z \in \operatorname{Per}(H)} \operatorname{Spec}(H ; z) .
$$

Recall that $\Gamma_{\omega}$ is either a discrete or a countable dense subset of $\mathbb{R}$.

LEMMA 2.2. $\operatorname{Spec}(H)$ is a measure zero subset of $\mathbb{R}$.

Proof. We first note that $\operatorname{Spec}(H ; z) \subset \mathbb{R}$ is a countable subset of $\mathbb{R}$ for each $z$. We consider the Poincaré return map in a tubular neighborhood of each $z \in \operatorname{Per}(H)$. More precisely, we choose a small neighborhood $V \subset M$ of $z(0)$. We identify $V$ with $2 n$-ball $B^{2 n}(\delta)$ with the point $z(0)$ identified with the center of the ball. Choose another ball neighborhood $V^{\prime}=B^{2 n}\left(\delta^{\prime}\right)$ with $\bar{V} \subset V^{\prime}$ such that the (first) Poincare return map denoted by

$$
R_{z}: V \rightarrow V^{\prime} ; p \mapsto \phi_{H}^{1}(p)
$$

is well-defined. We now define a continuous map from $V$ to the space of piecewise smooth maps from $S^{1} \cong \mathbb{R} / \mathbb{Z}$ on $M$ as follows: for each $p \in V$, we first follow the flow of $X_{H}$ and then follow from $R_{z}(p)$ to $p$ by the straight line under the identification of $V^{\prime}$ with $B^{2 n}\left(\delta^{\prime}\right)$. We reparameterize the domain of the loop by re-scaling it to be $[0,1]$.

We denote by $z_{p}$ the loop corresponding to $p \in V$ constructed as above, and by $V_{z} \subset \Omega_{0}(M)$ the image of the assignment $p \mapsto z_{p}$. Obviously $z_{p}$ is homotopic to $z$ and so any given disc $w$ bounding $z$ can be naturally continued to bound the loop $z_{p}$. We denote by $w_{p}$ the disc continued from $w$ and corresponding to $p \in V$. It can be easily checked that the function

$$
h: \pi^{-1}\left(V_{z}\right) \rightarrow \mathbb{R} ; \quad h\left(\left[z_{p}, w_{p}\right]\right):=\mathcal{A}_{H}\left(\left[z_{p}, w_{p}\right]\right)
$$

defines a smooth function on $\pi^{-1}\left(V_{z}\right)$ and its critical values comprise those of $\mathcal{A}_{H}$ near $\operatorname{Spec}(H ; z)$. This can be proven by writing $\mathcal{A}_{H}\left(\left[z_{p}, w_{p}\right]\right)$ explicitly and by a simple local calculation. Noting that $\pi^{-1}\left(V_{z}\right)$ is a finite dimensional (in fact, $2 n$ dimensional) manifold, Sard's theorem implies that the set of critical values is a measure zero subset in $\mathbb{R}$. Since a finite number of such tubular neighborhoods together with their complement cover $M, \operatorname{Spec}(H) \subset \mathbb{R}$ is a finite union of measure zero subset of $\mathbb{R}$ and so itself has measure zero.

For given $\phi \in \mathcal{H a m}(M, \omega)$, we denote by $H \mapsto \phi$ if $\phi_{H}^{1}=\phi$, and denote

$$
\mathcal{H}(\phi)=\{H \mid H \mapsto \phi\} .
$$


We say that two Hamiltonians $H$ and $K$ are equivalent if they are connected by one parameter family of Hamiltonians $\left\{F^{s}\right\}_{0 \leq s \leq 1}$ such that $F^{s} \mapsto \phi$ i.e.,

$$
\phi_{F^{s}}^{1}=\phi
$$

for all $s \in[0,1]$. We denote by $[H]$ the equivalence class of $H$. Then the universal covering space $\widetilde{\mathcal{H a m}}(M, \omega)$ of $\operatorname{Ham}(M, \omega)$ is realized by the set of such equivalence classes.

Let $F, G \mapsto \phi$ and denote

$$
f_{t}=\phi_{F}^{t}, g_{t}=\phi_{G}^{t}, \text { and } h_{t}=f_{t} \circ g_{t}^{-1} .
$$

Note that $h=\left\{h_{t}\right\}$ defines a loop based at the identity. Suppose $F \sim G$ so there exists a family $\left\{F^{s}\right\}_{0 \leq s \leq 1} \subset \mathcal{H}(\phi)$ with $F_{1}=F$ and $F_{0}=G$ and satisfying (2.3). In particular $h$ defines a contractible loop. If we denote $f_{t}^{s}=\phi_{F^{s}}^{t}$, this family provides a natural contraction of the loop $h$ to the identity through

$$
\widetilde{h}: s \mapsto f^{s} \circ g^{-1} ; \quad f^{s} \circ g^{-1}(t):=f_{t}^{s} \circ g_{t}^{-1} .
$$

which in turn provides a natural lifting of the action of the loop $h$ on $\Omega_{0}(M)$ to $\widetilde{\Omega}_{0}(M)$ which we define

$$
\tilde{h} \cdot[\gamma, w]=[h \gamma, \tilde{h} w]
$$

where $\tilde{h} w$ is the natural map from $D^{2}$ obtained from identifying $\widetilde{h}:[0,1] \times[0,1] \rightarrow$ $\operatorname{Ham}(M, \omega)$ as a map from $D^{2}$.

Even when $F$ is not homotopic to $G$ and so $h$ is not contractible, note that the (based) loop group $\Omega(\mathcal{H a m}(M, \omega), i d)$ naturally acts on the loop space $\Omega(M)$ by

$$
(h \cdot \gamma)(t)=h(t)(\gamma(t))
$$

where $h \in \Omega(\mathcal{H a m}(M, \omega))$ and $\gamma \in \Omega(M)$. An interesting consequence of Arnold's conjecture is that this action maps the particular component $\Omega_{0}(M) \subset \Omega(M)$ to itself (see e.g., [Lemma 2.2, Se]). Seidel [Lemma 2.4, Se] proves that this action (by a based loop) can be lifted to $\widetilde{\Omega}_{0}(M)$. In this paper, we will consider only the action by contractible loops in $\mathcal{H a m}(M, \omega)$.

We now study the behavior of the action spectrum Spec $H$ when $H$ varies. In particular, we would like to study the continuity property of certain critical values which are relevant to the uniform minimum point of the given quasi-autonomous Hamiltonian. For this purpose, we need to normalize the spectrum $\mathrm{Spec} H$. We will achieve this by restricting ourselves to $\mathcal{H}_{0}(\phi)$ the set of normalized Hamiltonians with $H \mapsto \phi$ by $\int_{M} H_{t} d \mu=0$ as in [Sc]. The following is proved in [Oh6] (see [Sc] for the symplectically aspherical case where the action fuctional is single-valued. In this case Schwarz [Sc] proved that the normalization works on $\operatorname{Ham}(M, \omega)$ not just on $\widetilde{\mathcal{H a m}}(M, \omega)$ as long as $F, G \mapsto \phi$, without assuming $F \sim G)$.

Proposition 2.3 [Theorem I, OH6]. Let $F, G \in \mathcal{H}_{0}(\phi)$ and $\mathcal{F}=\left\{F^{s}\right\}_{s \in[0,1]}$ be a path in $\mathcal{H}_{0}(\phi)$ such that $F^{0}=G$ and $F^{1}=F$. Denote $h_{t}^{s}=f_{t}^{s} \circ g_{t}^{-1}$ and $h^{s} \cdot[z, w]=\left[h^{s} \cdot z, \widetilde{h}^{s} \cdot w\right]$ for a $z \in \operatorname{Per}(G)$. Then the function $\chi:[0,1] \rightarrow \mathbb{R}$ defined by

$$
\chi(s)=A_{F^{s}}\left(\tilde{h}^{s} \cdot[z, w]\right)
$$


is constant. In particular, we have

$$
\operatorname{Spec}(G)=\operatorname{Spec}(F) .
$$

From now on, we will always assume that the Hamiltonian functions are normalized so that

$$
\int_{M} H_{t} d \mu=0
$$

\section{Floer homology with real filtration.}

3.1. Behavior of the filtration under the chain map. For each given generic $H: M \times S^{1} \rightarrow \mathbb{R}$, we consider the free $\mathbb{Q}$ vector space over

$$
\operatorname{Crit} \mathcal{A}_{H}=\left\{[z, w] \in \widetilde{\Omega}_{0}(M) \mid z \in \operatorname{Per}(H)\right\} .
$$

To be able to define the Floer boundary operator correctly, we need to complete this vector space downward with respect to the real filtration provided by the action $\mathcal{A}_{H}([z, w])$ of the element $[z, w]$ of (3.1). More precisely,

DEFINITION 3.1. We call the formal sum

$$
\beta=\sum_{[z, w] \in \operatorname{Crit} \mathcal{A}_{H}} a_{[z, w]}[z, w], a_{[z, w]} \in \mathbb{Q}
$$

a Novikov chain if there are only finitely many non-zero terms in the expression (3.2) above any given level of the action. We denote by $\widetilde{C F}(H)$ the set of Novikov chains.

Here, we put 'tilde' over $C F$ to distinguish this $\mathbb{Q}$ vector space with more standard Floer complex module over the Novikov ring in the literature. Note that this is an infinite dimensional $\mathbb{Q}$-vector space in general, unless $\pi_{2}(M)=0$. It appears that for the purpose of studying Hofer's geometry this set-up of the Floer homology with real filtration on the $\Gamma$-covering space $\widetilde{\Omega}_{0}(M)$ suits better than the more standard Floer homology on $\Omega_{0}(M)$ with the Novikov ring as its coefficient, although they provide equivalent descriptions.

Since we will frequently use the chain level property of various operators in the Floer theory for the study of changes of the action under the chain map, we briefly review the construction of the basic operators in the Floer homology theory [Fl2]. Let $J=\left\{J_{t}\right\}_{0 \leq t \leq 1}$ be a periodic family of compatible almost complex structures on $(M, \omega)$.

For each given pair $(J, H)$, we define the boundary operator

$$
\partial: \widetilde{C F}(H) \rightarrow \widetilde{C F}(H)
$$

considering the perturbed Cauchy-Riemann equation

$$
\left\{\begin{array}{l}
\frac{\partial u}{\partial \tau}+J\left(\frac{\partial u}{\partial t}-X_{H}(u)\right)=0 \\
\lim _{\tau \rightarrow-\infty} u(\tau)=z^{-}, \lim _{\tau \rightarrow \infty} u(\tau)=z^{+}
\end{array}\right.
$$

This equation, when lifted to $\widetilde{\Omega}_{0}(M)$, defines nothing but the negative gradient flow of $\mathcal{A}_{H}$ with respect to the $L^{2}$-metric on $\widetilde{\Omega}_{0}(M)$ induced by the metrics $g_{J_{t}}:=\omega\left(\cdot, J_{t} \cdot\right)$. 
For each given $\left[z^{-}, w^{-}\right]$and $\left[z^{+}, w^{+}\right]$, we define the moduli space $\mathcal{M}_{J}\left(\left[z^{-}, w^{-}\right],\left[z^{+}, w^{+}\right]\right)$ of solutions $u$ of (3.3) satisfying

$$
w^{-} \# u \sim w^{+}
$$

$\partial$ has degree -1 and satisfies $\partial \circ \partial=0$.

When we are given a family $(j, \mathcal{H})$ with $\mathcal{H}=\left\{H^{s}\right\}_{0 \leq s \leq 1}$ and $j=\left\{J^{s}\right\}_{0 \leq s \leq 1}$, the chain homomorphism

$$
h_{(j, \mathcal{H})}: \widetilde{C F}\left(J^{0}, H^{0}\right) \rightarrow \widetilde{C F}\left(J^{1}, H^{1}\right)
$$

is defined by the non-autonomous equation

$$
\left\{\begin{array}{l}
\frac{\partial u}{\partial \tau}+J^{\rho_{1}(\tau)}\left(\frac{\partial u}{\partial t}-X_{H^{\rho_{2}}(\tau)}(u)\right)=0 \\
\lim _{\tau \rightarrow-\infty} u(\tau)=z^{-}, \lim _{\tau \rightarrow \infty} u(\tau)=z^{+} .
\end{array}\right.
$$

where $\rho_{i}, i=1,2$ is functions of the type $\rho: \mathbb{R} \rightarrow[0,1]$,

$$
\begin{aligned}
& \rho(\tau)= \begin{cases}0 & \text { for } \tau \leq-R \\
1 & \text { for } \tau \geq R\end{cases} \\
& \rho^{\prime}(\tau) \geq 0
\end{aligned}
$$

for some $R>0 . h_{(j, \mathcal{H})}$ has degree 0 and satisfies

$$
\partial_{\left(J^{1}, H^{1}\right)} \circ h_{(j, \mathcal{H})}=h_{(j, \mathcal{H})} \circ \partial_{\left(J^{0}, H^{0}\right)} .
$$

Finally when we are given a homotopy $(\bar{j}, \overline{\mathcal{H}})$ of homotopies with $\bar{j}=\left\{j_{\kappa}\right\}$, $\overline{\mathcal{H}}=\left\{\mathcal{H}_{\kappa}\right\}$, consideration of the parameterized version of (3.5) for $0 \leq \kappa \leq 1$ defines the chain homotopy map

$$
\widetilde{H}: \widetilde{C F}\left(J^{0}, H^{0}\right) \rightarrow \widetilde{C F}\left(J^{1}, H^{1}\right)
$$

which has degree +1 and satisfies

$$
h_{\left(j_{1}, \mathcal{H}_{1}\right)}-h_{\left(j_{0}, \mathcal{H}_{0}\right)}=\partial_{\left(J^{1}, H^{1}\right)} \circ \widetilde{H}+\widetilde{H} \circ \partial_{\left(J^{0}, H^{0}\right)} .
$$

By now, construction of these maps using the relevant moduli spaces has been completed with the rational coefficient (See [FOn], [LT] and [Ru]). We will freely use this advanced machinery throughout the paper. However the main stream of the proof can be read independently of these papers once it is understood that the bubblingoff-spheres is a codimension two phenomenon, which is exactly what the advanced machinery establishes. Therefore we do not explicitly mention these technicalities in this paper, unless it is absolutely necessary.

The following upper estimate of the action change can be proven by the same argument as that of [Oh3]. Because this will be a crucial ingredient in our proof, we include its proof here for reader's convenience.

Proposition 3.2 [TheOrem 7.2, OH3]. Let $H, K$ be any Hamiltonian not necessarily non-degenerate and $j=\left\{J^{s}\right\}_{s \in[0,1]}$ be any given homotopy and $\mathcal{H}^{\text {lin }}=$ $\left\{H^{s}\right\}_{0 \leq s \leq 1}$ be the linear homotopy $H^{s}=(1-s) H+s K$. Suppose that (3.5) has a 
solution satisfying (3.4). Then we have the identity

$$
\begin{aligned}
\mathcal{A}_{F}\left(\left[z^{+}, w^{+}\right]\right) & -\mathcal{A}_{H}\left(\left[z^{-}, w^{-}\right]\right) \\
& =-\int\left|\frac{\partial u}{\partial \tau}\right|_{J^{\rho_{1}(\tau)}}^{2}-\int_{-\infty}^{\infty} \rho^{\prime}(\tau)(F(t, u(\tau, t))-H(t, u(\tau, t))) d t d \tau \\
& \leq-\int\left|\frac{\partial u}{\partial \tau}\right|_{J^{\rho_{1}(\tau)}}^{2}+\int_{0}^{1}-\min _{x \in M}\left(F_{t}-H_{t}\right) d t \\
& \leq \int_{0}^{1}-\min _{x \in M}\left(F_{t}-H_{t}\right) d t
\end{aligned}
$$

In particular, when $H$ and $K$ are nondegenerate, the canonical chain map

$$
h_{H K}^{\text {lin }}: \widetilde{C F}(J, H) \rightarrow \widetilde{C F}(J, K)
$$

restricts to

$$
h_{H K}^{l i n}: \widetilde{C F}^{(-\infty, a]}(J, H) \rightarrow \widetilde{C F}^{\left(-\infty, a-\int \min (K-H) d t\right]}(J, K)
$$

and so induces the homomorphism

$$
h_{H K}^{l i n}: \widetilde{H F}^{(-\infty, a]}(J, H) \rightarrow \widetilde{H F}^{\left(-\infty, a-\int \min (K-H) d t\right]}(J, K)
$$

Proof. We fix $J$ here. Let $\left[z^{+}, w^{+}\right] \in \widetilde{C F}(K)$ and $\left[z^{-}, w^{-}\right] \in \widetilde{C F}(H)$ be given. As argued in [Oh3], for any given solution $u$ of (3.5) and (3.4), we compute

$$
\mathcal{A}_{K}\left(\left[z^{+}, w^{+}\right]\right)-\mathcal{A}_{H}\left(\left[z^{-}, w^{-}\right]\right)=\int_{-\infty}^{\infty} \frac{d}{d \tau}\left(\mathcal{A}_{H^{\rho_{2}(\tau)}}(u(\tau)) d \tau\right.
$$

Here we have

$$
\frac{d}{d \tau}\left(\mathcal{A}_{H^{\rho_{2}(\tau)}}(u(\tau))=d \mathcal{A}_{H^{\rho_{2}(\tau)}}(u(\tau))\left(\frac{\partial u}{\partial \tau}\right)-\int_{0}^{1}\left(\frac{\partial H^{\rho_{2}(\tau)}}{\partial \tau}\right)(u, t) d t\right.
$$

However since $u$ satisfies (3.5), we have

$$
\begin{aligned}
d \mathcal{A}_{H^{\rho_{2}(\tau)}}(u(\tau))\left(\frac{\partial u}{\partial \tau}\right) & =\int_{0}^{1} \omega\left(\frac{\partial u}{\partial t}-X_{H^{\rho_{2}}(\tau)}(u), \frac{\partial u}{\partial \tau}\right) d t \\
& =-\int_{0}^{1}\left|\frac{\partial u}{\partial \tau}\right|_{J_{t}^{\rho_{1}(\tau)}}^{2}
\end{aligned}
$$

and

$$
\int_{0}^{1}\left(\frac{\partial H^{\rho_{2}(\tau)}}{\partial \tau}\right)(u, t) d t=-\int_{0}^{1} \rho_{2}^{\prime}(\tau)(K-H)(u, t) d t .
$$

The identity (3.6) follows by integrating (3.10) over $\tau$ after substituting (3.11), (3.12) into (3.10). (3.7) follows from (3.6) and the inequality $\rho_{2}^{\prime}(\tau) \geq 0$. (3.8) is obvious and (3.9) follows from (3.8) by the definition of the chain map. This finishes the proof. 
Proposition 3.3 [Lemma 4.3, Он3]. For a fixed $H$ and for a given one parameter family $\bar{J}=\left\{J^{s}\right\}_{s \in[0,1]}$, the natural chain map

$$
h_{\bar{J}}: \widetilde{C F}\left(J^{0}, H\right) \rightarrow \widetilde{C F}\left(J^{1}, H\right)
$$

respects the filtration.

Proof. A similar computation, this time using (3.2) and (3.3) with $H$ fixed, leads to

$$
\mathcal{A}_{H}\left(\left[z^{+}, w^{+}\right]\right)-\mathcal{A}_{H}\left(\left[z^{-}, w^{-}\right]\right)=-\int_{-\infty}^{\infty} \int_{0}^{1}\left|\frac{\partial u}{\partial \tau}\right|_{J \rho(\tau)}^{2} \leq 0 .
$$

We refer to the proof of [Lemma 4.3, Oh3] for complete details.

We would like to remark that there is also some upper estimate for the chain map over the general homotopy or for the chain homotopy maps. This general upper estimate is used in our construction of the spectral invariants in [Oh7].

3.2. The adiabatic homotopy and the adiabatic chain map. For our purpose of using the Floer theory in the study of Hofer's geometry, we also need to consider a family version of the Floer homology to keep track of the behavior of the action spectrum over one parameter family of Hamiltonians as in $\S 2$.

Let $\phi \in \mathcal{H a m}(M, \omega)$ and $\mathcal{F}=\left\{F^{s}\right\}_{s \in[0,1]}$ be a path in $\mathcal{H}(\phi)$. We normalize $F^{s}$ so that (2.5) (and so Proposition 2.3) holds. With this normalization, if $\operatorname{Spec}(G) \subset \mathbb{R}$ were isomorphic to $\Gamma_{\omega} \mathbb{Z}$ or $\{0\}$ like the case where $\pi_{2}(M)=0$ or more generally where $(M, \omega)$ is integral, the "adiabatic" homotopy

$$
h_{\mathcal{F}}^{a d b}: \widetilde{C F}(J, G) \rightarrow \widetilde{C F}(J, F)
$$

as defined in $[\mathrm{MO} 1,2]$ will induce an isomorphism

$$
h_{\mathcal{F}}^{a d b}: \widetilde{H F}^{(-\infty, a]}(J, G) \rightarrow \widetilde{H F}^{(-\infty, a]}(J, F)
$$

for any $a \in \mathbb{R}$. Since we will use this adiabatic homotopy in an essential way later, we carefully explain how it is constructed following the exposition from [MO1,2].

Suppose that there is a 'gap' in the spectrum $\operatorname{Spec}(G)=\operatorname{Spec}\left(F^{s}\right)$, i.e, that there is a positive number $\epsilon>0$ such that

$$
|\lambda-\mu| \geq \epsilon
$$

for all $\lambda \neq \mu \in \operatorname{Spec}(G)$.

Since $s \mapsto F^{s}$ is a smooth path, there exists some $\delta>0$ such that

$$
\left\|F^{u}-F^{s}\right\|_{C^{0}}<\frac{\epsilon}{3}
$$

for all $u, s \in[0,1]$ with $|u-s|<\delta$. We consider the partition

$$
I: 0=t_{0}<t_{1}<\cdots<t_{N}=1
$$

so that

$$
\left|t_{j}-t_{j+1}\right|<\delta \text { for all } j .
$$

By Proposition 3.2, the chain map

$$
h_{u s}^{l i n}: \widetilde{C F}\left(F^{u}\right) \rightarrow \widetilde{C F}\left(F^{s}\right)
$$


over the linear path

$$
\mathcal{L}: r \mapsto(1-r) F^{u}+r F^{s} ; r \in[0,1]
$$

restricts to

$$
h_{u s}^{l i n}: \widetilde{C F}^{(-\infty, \lambda]}\left(F^{u}\right) \rightarrow \widetilde{C F}^{\left(-\infty, \lambda+\frac{\epsilon}{3}\right]}\left(F^{s}\right)
$$

for any $u, s \in[0,1]$ with $|u-s|<\delta$. Similarly, we have

$$
h_{s u}^{l i n}: \widetilde{C F}^{\left(-\infty, \lambda^{\prime}\right]}\left(F^{s}\right) \rightarrow \widetilde{C F}^{\left(-\infty, \lambda^{\prime}+\frac{\epsilon}{3}\right]}\left(F^{u}\right)
$$

for any $\lambda^{\prime} \in \mathbb{R}$. Combining these two, we have the composition

$$
h_{s u}^{l i n} \circ h_{u s}^{l i n}: \widetilde{C F}^{(-\infty, \lambda]}\left(F^{u}\right) \rightarrow \widetilde{C F}^{\left(-\infty, \lambda+\frac{2 \epsilon}{3}\right]}\left(F^{u}\right) .
$$

By the condition (3.5) and the gap condition, all of these three maps in fact restrict to the same levels and induces homomorphisms

$$
\begin{aligned}
& h_{u s}^{l i n}: \widetilde{H F}^{(-\infty, \lambda]}\left(F^{u}\right) \rightarrow \widetilde{H F}^{(-\infty, \lambda]}\left(F^{s}\right) \\
& h_{s u}^{l i n}: \widetilde{H F}^{(-\infty, \lambda]}\left(F^{s}\right) \rightarrow \widetilde{H F}^{(-\infty, \lambda]}\left(F^{u}\right)
\end{aligned}
$$

and

$$
h_{s u}^{l i n} \circ h_{u s}^{l i n}: \widetilde{H F}^{(-\infty, \lambda]}\left(F^{u}\right) \rightarrow \widetilde{H F}^{(-\infty, \lambda]}\left(F^{u}\right),
$$

provided $\lambda$ is chosen sufficiently close to $\operatorname{Spec}(G)$. However, if we choose $\delta$ sufficiently small, we can also prove the identity

$$
h_{s u}^{l i n} \circ h_{u s}^{l i n}=h_{u u}(=\mathrm{id}) \text { on } \widetilde{H F}^{(-\infty, \lambda)}\left(F^{u}\right)
$$

which implies that (3.14) is an isomorphism for all $u, s$ with $|u-s|<\delta$. By repeating the above to $(u, s)=\left(t_{j}, t_{j+1}\right)$ for $j=0, \ldots, N-1$, we conclude that the composition

$$
h_{t_{j} t_{j-1}}^{l i n} \circ h_{t_{j-1} t_{j-2}}^{\text {lin }} \circ \cdots \circ h_{t_{1} t_{0}}^{l i n}: \widetilde{C F}(G) \rightarrow \widetilde{C F}\left(F^{t_{j}}\right)
$$

restricts to

$$
h_{t_{j} t_{j-1}}^{l i n} \circ \cdots \circ h_{t_{1} t_{0}}^{l i n}: \widetilde{C F}^{(-\infty, \lambda]}(G) \rightarrow \widetilde{C F}^{(-\infty, \lambda]}\left(F^{t_{j}}\right)
$$

for all $1 \leq j \leq N$, and so induces the composition

$$
h_{t_{j} t_{j-1}}^{l i n} \circ \cdots \circ h_{t_{1} t_{0}}^{l i n}: \widetilde{H F}^{(-\infty, \lambda]}(G) \rightarrow \widetilde{H F}^{(-\infty, \lambda]}\left(F^{t_{j}}\right)
$$

which becomes an isomorphism. In particular, we have the isomorphism

$$
h_{t_{N} t_{N-1}}^{l i n} \circ \cdots \circ h_{t_{1} t_{0}}^{l i n}: \widetilde{H F}^{(-\infty, \lambda]}(J, G) \rightarrow \widetilde{H F}^{(-\infty, \lambda]}(J, F)
$$

Definition 3.4. Let $I: 0<\epsilon_{1}<\epsilon_{2}<\cdots<\epsilon_{N}=1$ be a partition. We define its mesh, denoted as $\Delta_{I}$, by

$$
\Delta_{I}:=\max _{j}\left|t_{j+1}-t_{j}\right| .
$$

We call the associated piecewise continuous linear path $\mathcal{L}_{1} \# \mathcal{L}_{2} \# \cdots \# \mathcal{L}_{N-1}$ and the chain map (3.15) the adiabatic homotopy, denoted as $\mathcal{F}_{I}$, and the adiabatic chain map over the path $\mathcal{F}$. We denote

$$
h_{\mathcal{F}}^{I}=h_{t_{j} t_{j-1}}^{\operatorname{lin}} \circ \cdots \circ h_{t_{1} t_{0}}^{l i n}: \widetilde{C F}(G) \rightarrow \widetilde{C F}(F) .
$$


We define the mesh $\Delta\left(\mathcal{F}_{I}\right)$ of the adiabatic homotopy $\mathcal{F}_{I}$ along the path $\mathcal{F}$ to be

$$
\Delta\left(\mathcal{F}_{I}\right):=\max _{j=0, \cdots, N-1}\left\{\int_{0}^{1}-\min \left(F^{t_{j}}-F^{t_{j+1}}\right) d t, \int_{0}^{1} \max \left(F^{t_{j}}-F^{t_{j+1}}\right) d t\right\}
$$

We simply denote by $\mathcal{F}^{a d b}, h_{\mathcal{F}}^{a d b}$ when we do not specify the partition $I$. Note that the mesh of the adiabatic homotopy can be made arbitrarily small by making $\Delta_{I}$ small.

This adiabatic construction of homotopy in the chain level will be used in a crucial way to study the global case of the length minimizing property of geodesics, where the action spectrum is not necessarily fixed and does not have a 'gap' in general.

\section{4. $C^{2}$-small Hamiltonians and the local Floer complex.}

4.1. The local Floer complex. In this section, we consider $C^{2}$-small Hamiltonians $F$ and the subset $\Omega_{N_{\Delta}}(M)$ of loops $\gamma$ with $(\gamma(0), \gamma(t)) \in M \times M$ contained in a fixed Darboux neighborhood $N_{\Delta}$ of the diagonal $\Delta \subset M \times M$ for all $t \in[0,1]$. In particular, any periodic orbit $z$ of $X_{H}$ contained in $\Omega_{N_{\Delta}}(M)$ has a canonical isotopy class of contraction $w_{z}$. We will always use this convention $w_{z}$ whenever there is a canonical contraction of $z$ like in this case of small loops. This provides a canonical embedding of $\Omega_{N_{\Delta}}(M) \subset \widetilde{\Omega}_{0}(M)$ defined by

$$
z \rightarrow\left[z, w_{z}\right]
$$

We denote by $\mathcal{H}_{\delta}$ the set

$$
\mathcal{H}_{\delta}=\left\{F:[0,1] \times M \rightarrow \mathbb{R} \mid\|F\|_{C^{2}} \leq \delta \text { and } \int_{M} F_{t}=0 \text { for all } t\right\} .
$$

Imitating the construction from [Fl2] and [Oh1], we define

Definition 4.1. For any $(J, F) \in \mathcal{J}_{\omega}(M) \times \mathcal{H}_{\delta}$ and for the given Darboux neighborhood $N_{\Delta}$ of the diagonal $\Delta \subset M \times M$ such that

$$
\phi_{F}^{t}(\Delta) \subset \operatorname{Int} N_{\Delta},
$$

we define

$$
\mathcal{M}\left(J, F: N_{\Delta}\right)=\left\{u \in \mathcal{M}(J, F) \mid(u(\tau)(0), u(\tau)(t)) \in \operatorname{Int} N_{\Delta} \text { for all } \tau\right\} .
$$

Consider the evaluation map

$$
e v: \mathcal{M}\left(J, F: N_{\Delta}\right) \rightarrow \Omega_{N_{\Delta}} ; \quad e v(u)=u(0) .
$$

For each open subset $\mathcal{U} \subset M \times M$ with $\Delta \subset \mathcal{U} \subset M \times M$, we define the local Floer complex in $\Omega_{\mathcal{U}}$ by

$$
\mathcal{S}(J, F: \mathcal{U}):=\operatorname{ev}(\mathcal{M}(J, F: \mathcal{U})) \subset \Omega_{\mathcal{U}}
$$

We say $\mathcal{S}(J, F: \mathcal{U})$ is isolated in $\mathcal{U}$ if its closure is contained in the interior of $\overline{\Omega_{\mathcal{U}}}$.

The following can be proved by the same method as that of [F12] (See Proposition $3.2[\mathrm{Oh} 1])$, to which we refer readers for its proof. 
Proposition 4.2. If $\mathcal{S}(J, F: \mathcal{U})$ is isolated in $\mathcal{U}$, then for all $\left(J^{\prime}, F^{\prime}\right) C^{\infty}$-close enough to $(J, F)$ in the $C^{\infty}$-topology, $\mathcal{S}\left(J^{\prime}, F^{\prime}: \mathcal{U}\right)$ is also isolated in $\mathcal{U}$.

Using this proposition, we can define the local Floer homology, denoted by $H F(J, F$ : $\mathcal{U})$. Furthermore, the restriction of the action functional to the image of the embedding $\Omega_{N_{\Delta}}(M) \subset \widetilde{\Omega}_{0}(M)$ provides a filtration on the local Floer complex. Proof of the following proposition is standard combining the existing methods in the Floer theory (see $[\S 3$, Oh1]).

Proposition 4.3. Let $\mathcal{U}$ be as above and $F, F^{\prime} \in \mathcal{H}_{\delta}$. Assume that $\delta>0$ so small that (4.2) holds for $F, F^{\prime}$. Then there exists a canonical isomorphism, we have

$$
h_{(J: \mathcal{U})}: H F(J, F: \mathcal{U}) \rightarrow H F\left(J, F^{\prime}: \mathcal{U}\right)
$$

whose matrix elements are given by the number of solutions of (4.4) below whose images are contained in $\mathcal{U}$ :

$$
\left\{\begin{array}{l}
\frac{\partial u}{\partial \tau}+J\left(\frac{\partial u}{\partial t}-X_{H^{\rho(\tau)}}(u)\right)=0 \\
u(-\infty)=x \in C F(F: \mathcal{U}), \quad u(\infty)=y \in C F\left(F^{\prime}: \mathcal{U}\right) \\
w_{x} \# u \sim w_{y} .
\end{array}\right.
$$

Following [Oh1], we call thin trajectories the solutions of the Cauchy-Riemann equations defining the boundary map or the chain map whose images are contained in $\mathcal{U}$.

4.2. Fix $\phi_{G}^{1}$ versus $\Delta \cap$ graph $\phi_{G}^{1}$ : comparison of the two Floer homology. The main goal of this sub-section is to prove that when $G$ is a $C^{2}$-small quasi-autonomous Hamiltonian, the minimum point $x^{-}$, which corresponds to a (local) maximum point of $\mathcal{A}_{G}$ in the local Floer complex, is homologically essential in the local Floer complex. There does not seem to be a direct way of proving this in the context of the Floer theory of Hamiltonian diffeomorphisms. We will need to use the intersection theoretic version of the Floer theory of Lagrangian submanifolds between $\Delta$ and graph $\phi_{G}^{1}$ in the product $(M,-\omega) \times(M, \omega)$. This kind of comparison argument has been around among the experts in the Floer theory but never been rigorously carried out before. As we will see below, contrary to the conventional wisdom in the literature, this comparison does not work in the chain level but works only in the homology level.

We now compare the local Floer homology $H F(J, G: \mathcal{U})$ of $C^{2}$-small Hamiltonian $G$ and two versions of its intersection counterparts, one $H F_{-J \oplus J, 0}\left(\Delta\right.$, graph $\left.\phi_{G}^{1}: \mathcal{U}\right)$ and the other $H F_{-J \oplus\left(\phi_{G}\right)^{*} J, 0 \oplus G}(\Delta, \Delta: \mathcal{U})$. We will be especially keen to keep track of filtration changes.

First we note that the two Floer complexes $\mathcal{M}_{-J \oplus J, 0}\left(\Delta\right.$, graph $\left.\phi_{G}^{1}: \mathcal{U}\right)$ and $\mathcal{M}_{-J \oplus\left(\phi_{G}\right)^{*} J, 0 \oplus G}(\Delta, \Delta: \mathcal{U})$ are canonically isomorphic by the assignment

$$
(\gamma(t), \gamma(t)) \mapsto\left(\gamma(t),\left(\phi_{G}^{t}\right)^{-1}(\gamma)(t)\right) .
$$

and so the two Lagrangian intersection Floer homology are canonically isomorphic: Here the above two moduli spaces are the solutions sets of the following CauchyRiemann equations

$$
\left\{\begin{array}{l}
\frac{\partial U}{\partial \tau}+(-J \oplus J) \frac{\partial U}{\partial t}=0 \\
U(\tau, 0) \in \Delta, U(\tau, 1) \in \operatorname{graph} \phi_{G}^{1}
\end{array}\right.
$$


and

$$
\left\{\begin{array}{l}
\frac{\partial U}{\partial \tau}+\left(-J \oplus\left(\phi_{G}^{1}\right)^{*} J\right)\left(\frac{\partial U}{\partial t}-X_{0 \oplus G}(U)\right)=0 \\
U(\tau, 0) \in \Delta, U(\tau, 1) \in \Delta
\end{array}\right.
$$

respectively, where $U=\left(u_{1}, u_{2}\right): \mathbb{R} \times[0,1] \rightarrow M \times M$. The relevant action functionals for these cases are given by

$$
\mathcal{A}_{0}([\Gamma, W])=-\int W^{*}(-\omega \oplus \omega)
$$

on $\widetilde{\Omega}\left(\Delta, \operatorname{graph} \phi_{G}^{1}: M \times M\right)$ and

$$
\mathcal{A}_{0 \oplus G}([\Gamma, W])=\mathcal{A}_{0}(\Gamma, W)-\int_{0}^{1}(0 \oplus G)(\Gamma(t), t) d t
$$

on $\widetilde{\Omega}(\Delta, \Delta: M \times M)$ where we denote

$$
\Omega\left(\Delta, \operatorname{graph} \phi_{G}^{1}: M \times M\right)=\left\{\Gamma:[0,1] \rightarrow M \times M \mid \Gamma(0) \in \Delta, \Gamma(1) \in \operatorname{graph} \phi_{G}^{1}\right\}
$$

and similarly for $\Omega\left(\Delta\right.$, graph $\left.\phi_{G}^{1}: M \times M\right)$. Again the 'tilde' means the covering space which can be represented by the set of pairs $[\Gamma, W]$ in a similar way (see [ $\S 2$, FOOO] for the complete discussion on this set-up for the Lagrangian intersection Floer homology theory). The relations between the action functionals (4.5), (4.6) and (2.1) are evident and respects the filtration.

Next we will attempt to compare $H F(J, G ; \mathcal{U})$ and $H F_{-J \oplus J, 0 \oplus G}(\Delta, \Delta: \mathcal{U})$. Without loss of any generality, we will concern Hamiltonians $G$ such that $G \equiv 0$ near $t=0,1$, which one can always achieve by perturbing $G$ without changing its time-one map (See Lemma 5.2).

It turns out that there is no direct way of identifying the corresponding Floer complexes between the two.

As an intermediate case, we consider the Hamiltonian $G^{\prime}: M \times[0,1]$ defined by

$$
G^{\prime}(x, t)= \begin{cases}0 & \text { for } 0 \leq t \leq \frac{1}{2} \\ 2 G(x, 2 t) & \text { for } \frac{1}{2} \leq t \leq 1\end{cases}
$$

and the assignment

$$
\left(u_{0}, u_{1}\right) \in \mathcal{M}_{-J \oplus J, 0 \oplus G}(\Delta, \Delta: \mathcal{U}) \mapsto v \in \mathcal{M}\left(J, G^{\prime}: \mathcal{U}\right)
$$

with $v(\tau, t):=\overline{u_{0}} \# u_{1}(2 \tau, 2 t)$. Here the map $\overline{u_{0}} \# u_{1}:[0,2] \rightarrow M$ is the map defined by

$$
\overline{u_{0}} \# u_{1}(\tau, t)= \begin{cases}u_{0}(\tau, 1-t) & \text { for } 0 \leq t \leq 1 \\ u_{1}(\tau, t-1) & \text { for } 1 \leq t \leq 2\end{cases}
$$

is well-defined and continuous because

$$
\begin{aligned}
& \overline{u_{0}}(\tau, 1)=u_{0}(\tau, 0)=u_{1}(\tau, 0) \\
& u_{1}(\tau, 1)=u_{0}(\tau, 1)=\overline{u_{0}}(\tau, 0) .
\end{aligned}
$$

Furthermore near $t=0,1$, this is smooth (and so holomorphic) by the elliptic regularity since $G^{\prime}$ is smooth (Recall that we assume that $G \equiv 0$ near $t=0,1$. Conversely, any element $v \in \mathcal{M}\left(J, G^{\prime}: \mathcal{U}\right)$ can be written as the form of $\overline{u_{0}} \# u_{1}$ which is uniquely determined by $v$. This proves that (4.7) is a diffeomorphism from 
$\mathcal{M}_{-J \oplus J, 0 \oplus G}(\Delta, \Delta: \mathcal{U})$ to $\mathcal{M}\left(J, G^{\prime}: \mathcal{U}\right)$ which induces a filtration-preserving isomorphism between $H F_{-J \oplus J, 0 \oplus G}(\Delta, \Delta: \mathcal{U})$ and $H F\left(J, G^{\prime}: \mathcal{U}\right)$

Finally, we need to relate $H F(J, G: \mathcal{U})$ and $H F\left(J, G^{\prime}: \mathcal{U}\right)$. For this we note that $G$ and $G^{\prime}$ can be connected by a one-parameter family $\bar{G}=\left\{G^{s}\right\}_{0 \leq s \leq 1}$ with

$$
G^{s}(x, t):= \begin{cases}0 & \text { for } 0 \leq t \leq \frac{s}{2} \\ \frac{2}{1+s} G\left(x, \frac{2}{1+s} t\right) & \text { for } \frac{s}{2} \leq t \leq 1 .\end{cases}
$$

And we have

$$
\phi_{G^{s}}^{1}=\phi_{G}^{1} \quad \text { for all } s \in[0,1] .
$$

Noting that there are only finite number of periodic trajectories in $C F(G: \mathcal{U})$, the "adiabatic argument" explained in $\S 3$ indeed proves that the adiabatic homomorphism

$$
h_{J, \bar{G}}^{a d b}: C F\left(G^{\prime}: \mathcal{U}\right) \rightarrow C F(G: \mathcal{U})
$$

respects the filtration and so the induced homomorphism in its homology

$$
h_{\bar{G}}^{a d b}: H F\left(J, G^{\prime}: \mathcal{U}\right) \rightarrow H F(J, G: \mathcal{U})
$$

becomes a filtration-preserving isomorphism.

We note that $\mathcal{S}(J, 0: \mathcal{U})$ is isolated in $\mathcal{U}$. Therefore if follows from Proposition 3.1 that if $\|G\|_{C^{2}}$ and $\|K\|_{C^{2}}$ are sufficiently small, both $\mathcal{S}(J, G: \mathcal{U})$ and $\mathcal{S}(J, K: \mathcal{U})$ are also isolated in $\mathcal{U}$.

We now apply the above discussion to the $C^{2}$-small quasi-autonomous Hamiltonian $G$ to prove the following homological essentialness of the minimum points of $G$ in the local Floer homology. Recall from the remark in the beginning of $\S 2$ that the minimum of $G$ corresponds to the maximum of the action functional and vice versa. We quote the following from [Definition 13.2.F, Po2] for a formulation of the homological essentialness of the critical point

Definition 4.4. Let $(C, \partial)$ be a complex with a given basis $B=\left\{e_{1}, \cdots, e_{k}\right\}$. An element $e \in B$ is called homologically essential if for any sub-complex $K$ of $C$ such that

$$
K \subset \operatorname{Span}(B \backslash\{e\}),
$$

the induced map $H_{*}(K, \partial) \rightarrow H_{*}(C, \partial)$ is not surjective.

For example, any (local) maximum point of a Morse function on compact $M$ is homologically essential in its Morse homology complex (see e.g., [Corollary 13.2.H, Po2] for its proof).

Proposition 4.5.. Suppose that $\|G\|_{C^{2}}<\delta$ with $\delta$ so small that graph $\phi_{G}^{1} \subset \mathcal{U}$ lies in the given Darboux neighborhood of $\Delta \subset M \times M$. Suppose that $G$ is quasiautonomous with the unique maximum point $x^{+}$and minimum point $x^{-}$. Then the critical point $x^{-}$is homologically essential in $\mathcal{S}(J, G: \mathcal{U})$.

Proof. In the above discussion, we have shown that $\mathcal{M}\left(J, G^{\prime}: \mathcal{U}\right)$ is diffeomorphic to $\mathcal{M}_{-J \oplus J, 0 \oplus G}(\Delta, \Delta: \mathcal{U}) \cong \mathcal{M}_{(-J \oplus J), 0}\left(\Delta\right.$, graph $\left.\phi_{G}^{1}: \mathcal{U}\right)$. We will first show that the intersection point $\left(x^{-}, x^{-}\right)$is homologically essential in the latter Floer complex, which in turn will imply the homological essentialness of $x^{-}$in $\mathcal{S}\left(J, G^{\prime}: \mathcal{U}\right)$.

Identifying $\mathcal{U}$ with a neighborhood of the zero section of the cotangent bundle $T^{*} \Delta$, we denote by $J_{\Delta}$ the canonical almost complex structure on $T^{*} \Delta$ associated to 
the Levi-Civita connection of a given Riemannian metric on $\Delta$. Since the image of $\mathcal{M}_{(-J \oplus J, 0)}\left(\Delta\right.$, graph $\left.\phi_{G}^{1}: \mathcal{U}\right)$ is isolated in $\mathcal{U}$, we may perturb $-J \oplus J$ to $J^{\prime}$ in $\mathcal{U}$ so that $J^{\prime} \equiv J_{\Delta}$ near the boundary of $\overline{\mathcal{U}}$ and also that

$$
\mathcal{M}_{(-J \oplus J)}\left(\Delta, \text { graph } \phi_{G}^{1}: \mathcal{U}\right)=\mathcal{M}_{J^{\prime}}\left(\Delta, \operatorname{graph} \phi_{G}^{1}: \mathcal{U}\right) \text {. }
$$

We connect $J^{\prime}$ and $J_{\Delta}$ by a path $\widetilde{J}_{t}$ on $\mathcal{U}$ so that $\widetilde{J}_{t} \equiv J_{\Delta}$ near the boundary for all $t \in[0,1]$. Noting that $T^{*} \Delta$ is pseudo-convex with respect to $J_{\Delta}$, the two local Floer complexes $\mathcal{S}_{J^{\prime}}\left(\Delta\right.$, graph $\left.\phi_{G}^{1}: \mathcal{U}\right)$ and $\mathcal{S}_{J_{\Delta}}\left(\Delta\right.$, graph $\left.\phi_{G}^{1}: \mathcal{U}\right)$ can be connected by an isolated continuation in $\mathcal{U}$. Recall from Proposition 3.2 that this continuation preserves the filtration of the Floer homology.

On the other hand graph $\phi_{G}^{1}$ is diffeomorphic to graph $d S \subset \mathcal{U} \subset T^{*} \Delta$ for a generating function of the Lagrangian submanifold graph $\phi_{G}^{1} \subset T^{*} \Delta$, if $G$ is $C^{2}$ small. Moreover $x^{-}$corresponds to $\left(x^{-}, x^{-}\right)$which is the minimum point of the generating function $S: \Delta \rightarrow \mathbb{R}$. Since $M$ (and so $\Delta$ ) is assumed to be compact, $\left(x^{-}, x^{-}\right)$is homologically essential in the Morse homology of $-S$. On the other hand, $\mathcal{M}_{J_{\Delta}}(\Delta, \operatorname{graph}(-d S): \mathcal{U})$ is diffeomorphic to $\mathcal{M}^{\text {Morse }}(-S, g)$ (see [FOh] for its proof), where $J_{\Delta}$ is the almost complex structure on $T^{*} \Delta$ that is associated to the Levi-Civita connection of a chosen metric $g$ on $\Delta$. Therefore $\left(x^{-}, x^{-}\right)$is homologically essential in $\mathcal{M}_{J_{\Delta}}\left(\Delta\right.$, graph $\left.\phi_{G}^{1}: \mathcal{U}\right)$. Combining all these, we derive that the constant solution $x^{-}$is homologically essential in the local Floer complex $\mathcal{M}\left(J, G^{\prime}: \mathcal{U}\right)$.

By the uniqueness of the minimum points, under the chain isomorphism (4.8), the image $h_{\bar{G}}^{a d b}\left(x^{-}\right)$must involve $x^{-}$in its expression and so $x^{-}$is also homologically essential in $\mathcal{S}_{J_{\Delta}}(J, G: \mathcal{U})$. We refer readers to the proof of this kind of result in a more difficult context in $\S 7$.

5. Calculation. In this section, we start with the proof of Theorem $I$ in the introduction.

We consider the rescaled Hamiltonians

$$
\epsilon G^{\epsilon}=\epsilon G(\cdot, \epsilon t) \quad 0<\epsilon \leq 1 .
$$

and choose $\epsilon_{0}>0$ so small that it has no non-constant contractible periodic orbit for all $0<\epsilon \leq \epsilon_{0}$.

We first prove the following simple lemma.

LEMMA 5.1. Let $\left\{G_{i}\right\}$ be a sequence of smooth Hamiltonians such that $G_{i} \rightarrow G_{0}$ in $C^{0}$-topology and $\phi_{G_{i}} \rightarrow \phi_{G_{0}}$ in $C^{0}$-topology. If all $G_{i}$ are length minimizing over $[a, b]$, then so is $G_{0}$.

Proof. Suppose the contrary that there exists $F$ such that $F \sim G_{0}$, but $\|F\|<$ $\left\|G_{0}\right\|$. We choose $\delta>0$ with

$$
\|F\|<\left\|G_{0}\right\|-\delta
$$

Therefore

$$
\|F\| \leq\left\|G_{i}\right\|-\frac{1}{2} \delta
$$

for sufficiently large $i$. We consider the Hamiltonian $F_{i}$ defined by

$$
\begin{aligned}
F_{i} & :=\left(G_{i} \# \overline{G_{0}}\right) \# F \\
& =G_{i}-G_{0}\left(\phi_{G_{i}}^{t}\right)+F\left(\phi_{G_{0}}^{t} \circ\left(\phi_{G_{i}}^{t}\right)^{-1}\right)
\end{aligned}
$$


This generates the flow $\phi_{G_{i}}^{t} \circ\left(\phi_{G_{0}}^{t}\right)^{-1} \circ \phi_{F}^{t}$ and so $F_{i} \sim G_{i}$. This implies, by the hypothesis that $G_{i}$ are length minimizing over $[a, b]$, we have

$$
\left\|G_{i}\right\| \leq\left\|F_{i}\right\|
$$

and so

$$
\|F\| \leq\left\|F_{i}\right\|-\frac{1}{2} \delta
$$

for all sufficiently large $i$. However since $G_{i} \rightarrow G_{0}, \phi_{G_{i}} \rightarrow \phi_{G_{0}}$ by the hypotheses (and also so $\phi_{G_{0}} \circ\left(\phi_{G_{i}}\right)^{-1} \rightarrow i d$ ) in $C^{0}$-topology, we have $F_{i} \rightarrow F$ in $C^{0}$-topology. Therefore we have

$$
\lim _{i \rightarrow \infty}\left\|F_{i}\right\|=\|F\|
$$

which gives rise to a contradiction to (5.3).

Now, using the Floer homology theory, we would like to show

$$
\|G\| \leq\|F\|
$$

for any $F \sim G$ when the quasi-autonomous Hamiltonian $G$ satisfies the hypothesis that there is no non-constant contractible periodic orbits. However we need to take care of a problem before applying the Floer theory, that is, $G$ not being time-periodic. The following lemma will be important in this respect.

Lemma 5.2. Let $H$ be a given Hamiltonian $H: M \times[0,1] \rightarrow \mathbb{R}$ and $\phi=\phi_{H}^{1}$ be its time-one map. Then we can perturb $H$ so that the perturbed Hamiltonian $H^{\prime}$ has the properties

(1) $\phi_{H^{\prime}}^{1}=\phi_{H}^{1}$

(2) $H^{\prime} \equiv 0$ near $t=0$ and 1 and in particular $H^{\prime}$ is time periodic

(3) Both $\left|\int_{0}^{1} \max _{x}\left(H^{\prime}-H\right) d t\right|$ and $\left|\int_{0}^{1} \min _{x}\left(H^{\prime}-H\right) d t\right|$ can be made as small as we want

(4) If $H$ is quasi-autonomous, so is $H^{\prime}$.

(5) There is a canonical one-one correspondence between $\operatorname{Per}(H)$ and $\operatorname{Per}\left(H^{\prime}\right)$ with their actions fixed.

Furthermore, this modification is canonical with the "smallness" in (3) can be chosen uniformly over $H$ depending only on the $C^{0}$-norm of $H$.

Proof. We first reparameterize $\phi_{H}^{t}$ in the following way: We choose a smooth function $\zeta:[0,1] \rightarrow[0,1]$ such that

$$
\zeta(t)= \begin{cases}0 & \text { for } 0 \leq t \leq \frac{\epsilon}{2} \\ 1 & \text { for } 1-\frac{\epsilon}{2} \leq t \leq 1\end{cases}
$$

and

$$
\zeta^{\prime}(t) \geq 0 \text { for all } t \in[0,1]
$$

and consider the isotopy

$$
\psi^{t}:=\phi_{H}^{\zeta(t)} .
$$

It is easy to check that the Hamiltonian generating the isotopy $\left\{\psi^{t}\right\}_{0 \leq t \leq 1}$ is $H^{\prime}=$ $\left\{H_{t}^{\prime}\right\}_{0 \leq t \leq 1}$ with $H_{t}^{\prime}=\zeta^{\prime}(t) H_{\zeta(t)}$. By definition, it follows that $H^{\prime}$ satisfies (1) and (2). 
For (3), we compute

$$
\begin{aligned}
& \int_{0}^{1} \max _{x}\left(H^{\prime}-H\right) d t=\int_{0}^{1} \max _{x}\left(\zeta^{\prime}(t) H_{\zeta(t)}-H_{t}\right) d t \\
& \quad \leq \int_{0}^{1} \max _{x}\left(\zeta^{\prime}(t)\left(H_{\zeta(t)}-H_{t}\right)\right) d t+\int_{0}^{1} \max _{x}\left(\left(\zeta^{\prime}(t)-1\right) H_{t}\right) d t
\end{aligned}
$$

For the first term,

$$
\begin{aligned}
\int_{0}^{1} \max _{x} & \left(\zeta^{\prime}(t)\left(H_{\zeta(t)}-H_{t}\right)\right) d t=\int_{0}^{1} \zeta^{\prime}(t) \max _{x}\left(H_{\zeta(t)}-H_{t}\right) d t \\
& \leq \int_{0}^{1} \zeta^{\prime}(t) \max _{x, t}\left|H_{\zeta(t)}-H_{t}\right| d t=\max _{x, t}\left|H_{\zeta(t)}(x)-H_{t}(x)\right|
\end{aligned}
$$

which can be made arbitrarily small by choosing $\zeta$ so that $\|\zeta-t\|_{C^{0}}$ become sufficiently small. For the second term,

$$
\begin{aligned}
\int_{0}^{1} \max _{x}\left(\left(\zeta^{\prime}(t)-1\right) H_{t}\right) d t & \leq \int_{0}^{1}\left|\zeta^{\prime}(t)-1\right| d t \cdot \max _{x, t} H(x, t) \\
& \leq\|H\|_{C^{0}} \int_{0}^{1}\left|\zeta^{\prime}(t)-1\right| d t
\end{aligned}
$$

Again by appropriately choosing $\zeta$, we can make

$$
\int_{0}^{1}\left|\zeta^{\prime}(t)-1\right| d t
$$

as small as we want. Combining these two, we have verified $\left|\int_{0}^{1} \max _{x}\left(H^{\prime}-H\right) d t\right|$ can be made as small as we want. Similar consideration applies to $\left|\int_{0}^{1} \min _{x}\left(H^{\prime}-H\right) d t\right|$ and hence we have finished the proof of (3). (4) and naturality of this modification are evident from the construction. (5) follows from simple comparison of corresponding actions of periodic orbits.

We will always perform this canonical modification in the rest of the paper whenever we would like to consider the Cauchy-Riemann equation associated to the Hamiltonian $H$, when $H$ is not a one-periodic Hamiltonian.

Let $F$ be an arbitrary Hamiltonian with $F \sim G$. We want to prove $\|G\| \leq\|F\|$. Applying Lemma 5.2 to $G$ and $F$, we may assume that $G$ and $F$ are time one periodic, allowing small errors and then getting rid of them by taking the limit. We will postpone the proof of the following crucial existence result to the next sections.

From now on, we will always denote by $w_{y}$ the constant disc $y$ for each given constant periodic orbit $y$.

Proposition 5.3. Let $G$ be quasi-autonomous and $x^{-}$be a fixed minimum point of it. Suppose $F \sim G$. Let $k$ be a Morse function on $M$ and consider the linear homotopy

$$
L^{s}=(1-s) \epsilon k+s F \text {. }
$$


Then there exists $\epsilon_{1}$ such that for any $0<\epsilon \leq \epsilon_{1}$, the continuation equation

$$
\left\{\begin{array}{l}
\frac{\partial u}{\partial \tau}+J^{\rho_{1}(\tau)}\left(\frac{\partial u}{\partial t}-X_{L^{\rho_{2}(\tau)}}(u)\right)=0 \\
u(-\infty)=y, \quad u(\infty)=z \\
w_{y} \# u \sim w
\end{array}\right.
$$

has a solution for some $\left[y, w_{y}\right] \in \operatorname{Crit}\left(\mathcal{A}_{\epsilon k}\right)$ and for some $[z, w] \in \operatorname{Crit} \mathcal{A}_{F}$ with

$$
\mathcal{A}_{F}([z, w]) \geq \mathcal{A}_{F}\left(\left[z^{-}, w_{z^{-}}\right]\right)\left(=\int_{0}^{1}-\min G d t\right)
$$

where $\left[z^{-}, w_{z^{-}}\right]=\widetilde{h} \cdot\left[x^{-}, w_{x^{-}}\right]$with $\tilde{h}$ defined as in (2.4).

Assuming this proposition for the moment, we proceed with the proof of Theorem I. The following calculation is a slight modification used by Polterovich [Po2] in our context which will lead to the proof of Theorem I once we prove Proposition 5.3.

We compute

$$
\mathcal{A}_{F}([z, w])-\mathcal{A}_{\epsilon k}\left(\left[y^{-}, w_{y^{-}}\right]\right)=\int_{-\infty}^{\infty} \frac{d}{d \tau}\left\{\mathcal{A}_{L^{\rho_{2}(\tau)}}\left(u(\tau), w^{-} \# u(\tau)\right)\right\} d \tau
$$

We have

$$
\begin{aligned}
\frac{d}{d \tau}\left\{\mathcal{A}_{L^{\rho_{2}(\tau)}}(u(\tau),\right. & \left.\left.w^{+} \# u(\tau)\right)\right\}=d \mathcal{A}_{L^{\rho_{2}(\tau)}}\left(\frac{\partial u}{d \tau}\right)-\rho_{2}^{\prime}(\tau) \int_{0}^{1}(F-\epsilon k)(u(\tau)) d t \\
& =\int_{0}^{1} \omega\left(\frac{\partial u}{\partial t}-X_{L^{\rho_{2}(\tau)}}(u), \frac{\partial u}{\partial \tau}\right)-\rho_{2}^{\prime}(\tau) \int_{0}^{1}(F-\epsilon k)(u(\tau)) d t \\
& =-\int_{0}^{1}\left|\frac{\partial u}{\partial t}-X_{L^{\rho_{2}(\tau)}}(u)\right|_{J}^{2}-\rho_{2}^{\prime}(\tau) \int_{0}^{1}(F-\epsilon k)(u(\tau)) d t \\
& \leq-\rho_{2}^{\prime}(\tau) \int_{0}^{1} \min (F-\epsilon k) \leq-\rho_{2}^{\prime}(\tau)\left(\int_{0}^{1} \min F-\int_{0}^{1} \max \epsilon k\right)
\end{aligned}
$$

Therefore by integrating this over $\tau$ from $-\infty$ to $\infty$, we have

$$
\mathcal{A}_{F}([z, w])-\mathcal{A}_{\epsilon k}\left(\left[y^{-}, w_{y^{-}}\right]\right) \leq \int_{0}^{1}-\min F+\|\epsilon k\|
$$

On the other hand, we derive

$$
\mathcal{A}_{F}([z, w]) \geq \mathcal{A}_{F}\left(\left[z^{-}, w_{z^{-}}\right]\right)=\mathcal{A}_{G}\left(\left[x^{-}, w_{x^{-}}\right]\right)=\int_{0}^{1}-\min G d t
$$

from the normalization condition (2.5), (5.6) and from the fact that $x^{-}$is the fixed minimum point over $t$. Therefore we have

$$
\int_{0}^{1}-\min G d t \leq \int_{0}^{1}-\min F+\|\epsilon k\|+\mathcal{A}_{\epsilon k}\left(\left[y^{-}, w_{y^{-}}\right]\right) \leq \int_{0}^{1}-\min F+2 \epsilon\|k\|
$$

By letting $\epsilon \rightarrow 0$, we have proven

$$
\int_{0}^{1} \min (G) \geq \int_{0}^{1} \min F
$$


By considering $\bar{F}:=-F\left(\phi_{F}^{t}(x), t\right)$ and $\bar{G}$ which generate $\phi_{F}^{-1}$ and $\phi_{G}^{-1}$ respectively and noting that $\bar{G}$ is also quasi-autonomous, we also prove

$$
\int_{0}^{1} \min (-G) \geq \int_{0}^{1} \min (-F)
$$

which is equivalent to

$$
\int_{0}^{1} \max (G) \leq \int_{0}^{1} \max F
$$

Combining (5.7) and (5.8), we have proved

$$
\|G\| \leq\|F\| \text {. }
$$

This will finish the proof of Theorem I up to the proof of Proposition 5.3.

6. Handle sliding lemma. In this section, we study an important ingredient in our proof, the Floer theoretic version of the 'handle sliding' lemma. equation

Let $H$ be any time periodic Hamiltonian and consider the Cauchy- Riemann

$$
\frac{\partial u}{\partial \tau}+J\left(\frac{\partial u}{\partial t}-X_{H(u)}(u)\right)=0
$$

for generic $J$. We call a solution $u$ trivial if it is $\tau$-independent, i.e., stationary. We define

$$
\begin{gathered}
A_{(J, H)}:=\inf \left\{\int\left|\frac{\partial u}{\partial \tau}\right|_{J}^{2} \mid u \text { satisfies (6.1) for some } \varepsilon \in[0,1]\right. \\
\text { and is not trivial }\} .
\end{gathered}
$$

The positivity of $A_{(J, H)}$ is an easy consequence of Gromov compactness type theorem, whose proof we omit.

We will need a family version of $A_{(J, H)}$. When there does not occur bifurcation of periodic orbits, one can define this to be

$$
A_{(j, \mathcal{H})}=\inf _{0 \leq s \leq 1} A_{\left(J^{s}, H^{s}\right)}
$$

However when there does occur bifurcation of periodic orbits, $A_{(j, \mathcal{H})}$ could be zero, which forces us to look at another positive constant the definition of which should be given more subtly to make it suitable for our purpose. In introducing this constant, we exploit the fact that only the index zero solutions of Floer's continuity equation (3.5) or (6.9) below are used in the definition of Floer's chain homotopy map.

We first recall that for a generic one parameter family $\{H(s)\}_{0 \leq s \leq 1}$, there are only finite number of points $\operatorname{Sing}=\left\{s_{1}, s_{2}, \cdots, s_{k_{1}}\right\} \subset[0,1]$ where there occurs either birth-death or death-birth type of bifurcation of periodic orbits (see [Lee] for a detailed proof of this). Furthermore at each such $s_{j}$, there is exactly one bifurcation orbit $z_{j}$ of $\dot{x}=X_{H\left(s_{j}\right)}(x)$ for which there is a continuous family of the pair $z^{+}(s), z^{-}(s)$ of periodic orbits of $\dot{x}=X_{H(s)}(x)$ for $\left|s-s_{j}\right|<\delta, \delta$ sufficiently small such that

(1) $z^{ \pm}(s) \rightarrow z_{j}$ as $s \rightarrow s_{j}$,

(2) the Conley-Zehnder indices satisfy

$$
\mu\left(\left[z^{+}, w^{+}\right]\right)=\mu\left(\left[z^{-}, w^{-}\right]\right)+1
$$


where $w^{+} \sim w^{-} \# u$ for $u$ a canonical 'short' cylinder between $z^{+}$and $z^{-}$. This latter condition makes sense because $z^{+}$and $z^{-}$are close when $\delta$ is sufficiently small.

We now prove the following important lemma

Lemma 6.1. Let $\{H(s)\}$ be a generic one parameter family as above. For each $s \in[0,1] \backslash$ Sing, we define

$$
A_{\left(J^{s}, H^{s}\right)}^{0}=\inf \left\{\int\left|\frac{\partial u}{\partial \tau}\right|_{J^{s}}^{2} \mid u \text { satisfies (6.1), not trivial and Index } u=0\right\}
$$

and

$$
A_{(j, \mathcal{H})}^{r e g, 0}=\inf _{s \in[0,1] \backslash \operatorname{Sing}} A_{\left(J^{s}, H^{s}\right)}^{0}
$$

Then $A_{(j, \mathcal{H})}^{r e g, 0}$ is strictly positive.

Proof. Suppose the contrary that $A_{(j, \mathcal{H})}^{r e g, 0}=0$, i.e., that there exists a sequence $r_{k} \in[0,1] \backslash \operatorname{Sing}$ with $r_{k} \rightarrow r_{\infty} \in(0,1)$ and $u_{j}$ solutions of $(6.1)$ for $\left(J^{r_{k}}, H^{r_{k}}\right)$ such that

$$
\int\left|\frac{\partial u_{k}}{\partial \tau}\right|_{J^{r_{k}}}^{2} \rightarrow 0, \quad \text { Index } u_{k}=0
$$

Then we must have, by choosing a subsequence if necessary,

$$
r_{\infty} \in \operatorname{Sing}
$$

and a bifurcation orbit $z_{\infty}$ of $\dot{x}=X_{H^{r_{\infty}}}(x)$ such that $u_{k} \rightarrow z_{\infty}$ uniformly and so

$$
u_{k}(\infty), u_{k}(-\infty) \rightarrow z_{\infty}
$$

Since $u_{k}( \pm \infty)$ are solutions of $\dot{x}=X_{H^{r_{k}}}(x)$, they must be the pair described in (1) right above (6.4) and hence

$$
\text { Index }\left(u_{k}\right)=\mu\left(\left[z^{+}\left(r_{k}\right), w^{+}\left(r_{k}\right)\right]\right)-\mu\left(\left[z^{-}\left(r_{k}\right), w^{-}\left(r_{k}\right)\right]=1 .\right.
$$

But this contradicts to the index condition in (6.6) which finishes the proof.

Again for a generic choice of $\left\{H^{s}\right\}$, we may assume that there are only finitely many points $t_{i} \in[0,1] \backslash \operatorname{Sing}$ with $i=1, \cdots, k_{2}$ at which (6.1) has exactly one nontrivial solution $u_{t_{i}}$ that has the Fredholm index 0 . (See [Fl1] for this kind of the generic argument.) We denote

$$
\mathcal{N} t=\left\{t_{i}\right\}_{i=1, \cdots, k_{2}} \subset[0,1] \backslash \operatorname{Sing} .
$$

Next we define

$$
A_{(j, \mathcal{H})}^{s i n g}=\min _{k}\left\{A_{\left(J^{\left.s_{k}, H^{s_{k}}\right)}\right.} \mid s_{k} \in \operatorname{Sing}\right\}
$$

which is again positive by Gromov type compactness theorem. Now we have the following crucial definition of a family version of the constant $A_{(J, H)}$ suitable for our purpose.

DeFinition 6.2. We define

$$
A_{(j, \mathcal{H})}^{0}=\min \left\{A_{(j, \mathcal{H})}^{r e g, 0}, A_{(j, \mathcal{H})}^{\text {sing }}\right\}>0 .
$$

The following proposition is an important ingredient of our proof. 
Proposition 6.3. (HANDle Sliding LEMMA). Let $j=\left\{J^{\eta}\right\}$ be a (two parameter) family of almost complex structures and $\{H(\eta)\}_{0 \leq \eta \leq 1}$ be a generic family of Hamiltonians. Let $A_{(j, \mathcal{H})}^{0}>0$ be the constant defined in Definition 6.2 and let $\eta_{1}, \eta_{2} \in[0,1]$. Then there exists $\delta_{0}>0$ such that if $\left|\eta_{1}-\eta_{2}\right|<\delta$, any finite energy solution $u$ with

$$
\text { Index } u=0
$$

of

$$
\frac{\partial u}{\partial \tau}+J^{\rho(\tau)}\left(\frac{\partial u}{\partial t}-X_{H^{\rho(\tau)}}(u)\right)=0
$$

must either satisfy

$$
\int\left|\frac{\partial u}{\partial \tau}\right|_{J \rho(\tau)}^{2} \leq \varepsilon(\delta)
$$

or

$$
\int\left|\frac{\partial u}{\partial \tau}\right|_{J \rho(\tau)}^{2} \geq A_{(j, \mathcal{H})}^{0}-\varepsilon(\delta)
$$

where for $\varepsilon(\delta) \rightarrow 0$ as $0<\delta \rightarrow 0$, provided $\delta \leq \delta_{0}$. Here $H^{s}$ is the linear path $H^{s}=(1-s) H\left(\eta_{1}\right)+s H\left(\eta_{2}\right)$ and $\rho$ is the standard function defined as before.

We call a solution $u$ of (6.9) very short if it satisfies (6.10), and long if it satisfies (6.11). We can phrase the content of this proposition as "Any short path is indeed very short".

Proof of Proposition 6.3. We prove this by contradiction. Suppose the contrary that there exists some $\varepsilon>0, \eta_{1}$ and $\eta_{j}$ with $\eta_{j} \rightarrow \eta_{1}$ as $j \rightarrow \infty$, and solutions $u_{j}$ that satisfy (6.8) and

$$
\frac{\partial u_{j}}{\partial \tau}+J^{\rho(\tau)}\left(\frac{\partial u_{j}}{\partial t}-X_{H_{\rho(\tau)}}\left(u_{j}\right)\right)=0
$$

but

$$
\varepsilon<\int\left|\frac{\partial u_{j}}{\partial \tau}\right|_{J \rho(\tau)}^{2}<A_{(j, \mathcal{H})}^{0}-\varepsilon
$$

In particular, the right half of (6.13) implies the uniform bound on the energy of $u_{j}$. As $j \rightarrow \infty$, the equation (6.12) converges to (6.1) with $H=H\left(\eta_{1}\right)$. By Gromov type compactness theorem, we have a cusp curve

$$
u_{\infty}=\sum_{k} u_{\infty, k}
$$

in the limit of a subsequence where each $u_{\infty, k}$ is a solution of (6.1) with $H=H\left(\eta_{1}\right)$. We also have

$$
\lim _{j} \int\left|\frac{\partial u_{j}}{\partial \tau}\right|_{J \rho(\tau)}^{2}=\sum_{k} \int\left|\frac{\partial u_{\infty, k}}{\partial \tau}\right|_{J \rho(\tau)}^{2} .
$$

On the other hand the left half of (6.13) implies that at least one of $u_{\infty, k}$ is not trivial.

Now we consider three cases separately: the first is the one where $\eta_{1} \in \operatorname{Sing}$ and the second where $\eta_{1} \in \mathcal{N} t$ and the rest where $\eta_{1} \in[0,1] \backslash(\operatorname{Sing} \cup \mathcal{N} t)$. When $\eta_{1} \in \operatorname{Sing}$, we must have

$$
\lim _{j} \int\left|\frac{\partial u_{j}}{\partial \tau}\right|_{J \rho(\tau)}^{2} \geq A_{\left(J, H\left(\eta_{1}\right)\right)}^{\operatorname{sing}} \geq A_{(j, \mathcal{H})}^{0}
$$


which gives rise to a contradiction to (6.13) if $j$ is sufficiently large. On the other hand, if $\eta_{1} \in \mathcal{N} t$, the cusp curve must contain one component $u_{\infty}$ that has Index 0 and is non-constant. Again the right hand side of (6.13) prevents this from happening. Finally when $\eta_{1} \in[0,1] \backslash(\operatorname{Sing} \cup \mathcal{N} t)$, the index condition Index $u_{j}=0$ and the transversality condition imply that all the components $u_{\infty, k}$ must be constant which again contradicts to LHS of (6.13) if $j$ is sufficiently large. This finishes the proof of the proposition.

An immediate corollary of this is the following estimate on the action.

Corollary 6.4. Let $j, \mathcal{H}$ and $\delta_{0}$ as in Proposition 6.3. Suppose $0<\delta \leq \delta_{0}$. If $u$ is very short, then we have the lower estimate

$$
-\varepsilon(\delta)+\int_{0}^{1}-\max _{x}\left(H\left(\eta_{2}\right)-H\left(\eta_{1}\right)\right) d t \leq \mathcal{A}_{H\left(\eta_{2}\right)}(u(+\infty))-\mathcal{A}_{H\left(\eta_{1}\right)}(u(-\infty))
$$

and so combined with the upper estimate (3.6), we have

$$
\begin{aligned}
-\varepsilon(\delta)+\int_{0}^{1}-\max _{x}\left(H\left(\eta_{2}\right)-H\left(\eta_{1}\right)\right) d t & \leq \mathcal{A}_{H\left(\eta_{2}\right)}(u(+\infty))-\mathcal{A}_{H\left(\eta_{1}\right)}(u(-\infty)) \\
& \leq \int_{0}^{1}-\min _{x}\left(H\left(\eta_{2}\right)-H\left(\eta_{1}\right)\right) d t
\end{aligned}
$$

If $u$ is not very short and so must be long, then we have the improved upper estimate

$$
\mathcal{A}_{H\left(\eta_{2}\right)}(u(+\infty))-\mathcal{A}_{H\left(\eta_{1}\right)}(u(-\infty)) \leq-A_{(j, \mathcal{H})}+\varepsilon+\int_{0}^{1}-\min _{x}\left(H\left(\eta_{2}\right)-H\left(\eta_{1}\right)\right) d t
$$

Proof. A straightforward computation as in the proof of Proposition 3.2 leads to the following general identity

$$
\begin{aligned}
\mathcal{A}_{H\left(\epsilon_{2}\right)}(u(+\infty)) & -\mathcal{A}_{H\left(\epsilon_{1}\right)}(u(-\infty)) \\
& =-\int\left|\frac{\partial u}{\partial \tau}\right|_{J^{\rho(\tau)}}^{2}-\int_{-\infty}^{\infty} \rho^{\prime}(\tau) \int_{0}^{1}\left(H\left(\epsilon_{2}\right)-H\left(\epsilon_{1}\right)\right)(u(\tau)) d t d \tau .
\end{aligned}
$$

Corollary 6.4 immediately follows from this and Proposition 6.3.

We will apply the above handle sliding lemma and its corollary to the adiabatic paths in the next section.

7. Non-pushing down lemma and the existence. In this section, we will assume the main hypothesis. This is the only section where we use the hypothesis. All the materials in the other sections are valid in arbitrary compact symplectic manifolds.

Hypothesis. Assume one of the following two cases:

(1) either $(M, \omega)$ is weakly exact, i.e., $\left.\omega\right|_{\pi_{2}(M)}=0$ or

(2) $H$ is autonomous on arbitrary $(M, \omega)$

In the beginning, we will approach both cases in the general setting of quasiautonomous cases on arbitrary $(M, \omega)$ and then explain how non-existence of quantum contributions enter our proof of the Non-pushing down lemma. 
Definition 7.1. Let $H: M \times[0,1] \rightarrow \mathbb{R}$ be a Hamiltonian which is not necessarily time-periodic and $\phi_{H}^{t}$ be its Hamiltonian flow.

(1) We call a point $p \in M$ a time $T$ periodic point if $\phi_{H}^{T}(p)=p$. We call $t \in[0, T] \mapsto \phi_{H}^{t}(p)$ a contractible time $T$-periodic orbit if it is contractible.

(2) When $H$ has a fixed critical point $p$ over $t \in[0, T]$, we call $p$ over-twisted as a time $T$-periodic orbit if its linearized flow $d \phi_{H}^{t}(p) ; t \in[0, T]$ on $T_{p} M$ has a closed trajectory of period less than $T$.

The remaining section will be occupied by the proof of the following result (Theorem I in the introduction).

TheOREM 7.2. We assume one of the two cases in the Hypothesis. Suppose that the quasi-autonomous Hamiltonian $G$ satisfies

(i) $\phi_{G}^{t}$ has no non-constant contractible periodic orbit of period less than one,

(ii) it has at least one fixed minimum and one fixed maximum which are not overtwisted.

Then the Hamiltonian path $\phi_{G}^{t}, 0 \leq t \leq 1$ is length minimizing in its homotopy class with fixed ends.

REMARK 7.3. (1) From our proof, it will be clear that the proof of Theorem 7.2 is stable under the $C^{2}$-small perturbation of the Hamiltonian and so allows sufficiently $C^{1}$-small non-constant contractible periodic orbits. It is rather awkward to state how small the perturbation can be, though.

(2) Considering $\epsilon G^{\epsilon}$ with $\epsilon<1$ but arbitrarily close to 1 and applying Lemma 5.2 , we may assume the stronger assumption "period less than equal to 1 " instead of "period less than 1" in both (1) and (2) in the hypotheses of the theorem. We will assume this stronger assumption in the proof.

We consider the reparameterized Hamiltonians $\epsilon \in\left[\epsilon_{0}, 1\right] \mapsto \epsilon G^{\epsilon}$. The assumption (i) implies that there is no appearance of non-constant contractible periodic orbit as $\epsilon$ moves from $\epsilon_{0}$ to 1 . The only possible bifurcation is by that of critical points of $\epsilon G^{\epsilon}$. This proves

LEMma 7.4. Suppose $G$ satisfies the above. Then for each $0<\epsilon \leq 1$, there is one-one correspondence between the set of contractible solutions and the set of points $x \in M$ such that

$$
d G_{t}(x)=0 \text { for all } 0<t \leq \epsilon
$$

Definition 7.5. We call a point $x[0, \epsilon]$-critical point of $G$ if $x$ satisfies (7.1). We denote by

$$
\operatorname{Crit}_{0}^{\epsilon}(G)
$$

the set of $[0, \epsilon]$-critical points of $G$.

It follows from Lemma 7.4 that for any $\epsilon^{\prime}>\epsilon \geq \epsilon_{0}$ there is a canonical injection

$$
i_{\epsilon^{\prime} \epsilon}: \operatorname{Crit}\left(\mathcal{A}_{\epsilon^{\prime} G^{\epsilon^{\prime}}}\right) \rightarrow \operatorname{Crit}\left(\mathcal{A}_{\epsilon G^{\epsilon}}\right) \hookrightarrow \operatorname{Crit}\left(\mathcal{A}_{\epsilon_{0} G^{\epsilon_{0}}}\right)
$$

and that there is a canonical one-one correspondence between the set of $[0, \epsilon]$-critical points of $G$ and that of critical points of $\mathcal{A}_{\epsilon G^{\epsilon}}$ which are of the type $\left[x, w_{x}\right]$. From this description of Crit $\mathcal{A}_{\epsilon G^{\epsilon}}$, it follows that there does not emerge any new critical points of $\mathcal{A}_{\epsilon G^{\epsilon}}$ as $\epsilon$ moves from $\epsilon_{0}$ to 1 . 
For any $[0, \epsilon]$-critical point $x$ of $G$, we have

$$
\mathcal{A}_{\epsilon G^{\epsilon}}\left(\left[x, w_{x}\right]\right)=-\int_{0}^{1} \epsilon G(x, \epsilon s) d s=-\int_{0}^{\epsilon} G(x, t) d t .
$$

We denote

$$
\gamma_{x}(\epsilon):=-\int_{0}^{\epsilon} G(x, t) d t
$$

and

$$
\gamma^{ \pm}(\epsilon)=-\int_{0}^{\epsilon} G\left(x^{\mp}, t\right) d t
$$

Using Lemma 5.1 and 5.2 and the conditions (i) and (ii) in the statement of Theorem 7.2 , by adding a small bump function around $x^{-}$, we may assume, without loss of any generality, that $x^{-}$is the unique minimum point of $G_{t}$ for each $t \in[0,1]$ and that there is a 'gap' between $-G\left(x^{-}, t\right)$ and $-G(x, t)$

$$
-G\left(x^{-}, t\right)+G(x, t)>\delta_{1}
$$

for all $t \in[0,1]$ for any $x \neq x^{-} \in \operatorname{Crit}{ }_{0}^{\eta}(G)$. Similar statement holds for the maximum point $x^{+}$. We will fix $\delta_{1}>0$ later in (7.15). This implies that for any $\eta \geq \epsilon_{0}$ we have

$$
\mathcal{A}_{\eta G^{\eta}}\left(\left[x^{-}, w_{x^{-}}\right]\right)-\mathcal{A}_{\eta G^{\eta}}\left(\left[x, w_{x}\right]\right)=\gamma^{+}(\eta)-\gamma_{x}(\eta)>\eta \delta_{1} \geq \epsilon_{0} \delta_{1}
$$

for any $[0, \eta]$-critical point $x \neq x^{-}$of $G$.

For the proof of Theorem 7.2, it will be enough to prove Proposition 5.3. The rest of this section will be occupied by its proof. We recall that we considered the linear homotopy $\mathcal{L}=\left\{L^{s}\right\}$,

$$
L^{s}=(1-s) \epsilon k+s F .
$$

and then studied the continuation equation

$$
\left\{\begin{array}{l}
\frac{\partial u}{\partial \tau}+J\left(\frac{\partial u}{\partial t}-X_{L^{\rho(\tau)}}(u)\right)=0 \\
u(-\infty)=y^{-} \in \operatorname{Crit}(K), \quad u(\infty)=z \\
y^{-} \# u \sim w .
\end{array}\right.
$$

Using Lemma 5.2, after the preliminary perturbation of $G$, we may assume that there are only finitely many constant periodic solutions of $\dot{x}=X_{G}(x)$.

We will construct a solution of the equation (7.6) in four steps: First by considering the linear homotopy

$$
\mathcal{K}: \epsilon k \mapsto \epsilon_{0} G^{\epsilon_{0}},
$$

we construct a cycle $\alpha \in\left(\widetilde{C F}\left(\epsilon_{0} G^{\epsilon_{0}}\right), \partial_{J, \epsilon_{0} G^{\epsilon_{0}}}\right)$ with its Floer homology class $[\alpha]$ being the fundamental class $[M] \in H_{2 n}(M) \subset F H_{*}(M)$. This can be realized by a linear combination of the form

$$
\alpha=\left[x^{-}, w_{x^{-}}\right]+\sum_{j} a_{j}\left[x_{j}, w_{x_{j}}\right], \quad a_{j} \in \mathbb{Q}
$$

where $x_{j}$ 's are the uniform critical points of $G_{t}$ over $t \in\left[0, \epsilon_{0}\right]$. This is an immediate consequence of the homological essentialness (Proposition 4.4) of $x^{-}$in the local Floer complex $C F\left(\epsilon G^{\epsilon}: \mathcal{U}\right)$, and of the Hypothesis above, which implies that there is no quantum contribution for the Floer boundary operator for the $C^{2}$-small Hamiltonians 
in either case. (See the proof of Proposition 7.11 below for the explanation for the case (2) of autonomous Hamiltonians).

Secondly we consider the homotopy

$$
\mathcal{G}: \eta \mapsto\left\{\eta G^{\eta}\right\}, \quad \eta \in\left[\epsilon_{0}, 1\right]
$$

from $\epsilon_{0} G^{\epsilon_{0}}$ to $G$. This step proves that the Novikov cycle $\alpha_{G}$ of $G$ transferred from $\alpha$ via the adiabatic homotopy along $\mathcal{G}$ satisfies the Non-pushing down lemma, i.e, cannot be pushed down by the Cauchy-Riemann flow of $G$. The proof heavily relies on the Hypothesis.

Thirdly we consider the homotopy

$$
\mathcal{F}: s \mapsto\left\{F^{s}\right\}, \quad s \in[0,1]
$$

from $G$ to $F$ which is provided by the definition $G \sim F$. Again this step proves that the Novikov cycle of $F$ transferred from $\alpha_{G}$ via the adiabatic homotopy along $\mathcal{F}$ cannot be pushed down by the Cauchy-Riemann flow of $F$. However its proof does not use the Hypothesis but uses only the fact $G \sim F$ and so the arguments used in this step can be applied in general.

Finally, we glue the homotopies $\mathcal{K}, \mathcal{G}$ and $\mathcal{F}$ and deform the glued homotopy $\mathcal{K} \#_{R_{1}} \mathcal{G} \#_{R_{2}} \mathcal{F}$ to the linear homotopy

$$
\mathcal{L}: s \mapsto(1-s) \epsilon_{0} G^{\epsilon_{0}}+s G .
$$

The arguments in this step are independent of the Hypothesis. In the rest of this section, we will carry out these steps.

Step I; from $\epsilon k \rightarrow \epsilon_{0} G^{\epsilon_{0}}$

To carry out the first step, it is essential to further analyze the general structure of the boundary operator for the $C^{2}$-small Hamiltonians (not necessarily quasiautonomous) like $\epsilon G^{\epsilon}$ of $\epsilon$ sufficiently small. This will be carried out following the argument used in $[\S 3, \mathrm{Oh} 1]$.

For each time independent $J_{0}$, we consider the quantity

$$
A=A\left(J_{0}, \omega: M\right):=\inf \left\{\int v^{*} \omega \mid v: S^{2} \rightarrow M, \bar{\partial}_{J_{0}} v=0, v \text { non-constant }\right\} .
$$

We choose $\epsilon>0$ so small and in particular so that $\left\|\epsilon G^{\epsilon}\right\|<\frac{1}{2} A\left(J_{0}, \omega: M\right)$.

We now state the following proposition, which is the analog of [Proposition 4.1, Oh1] to which we refer readers for its proof (see also [Appendix 1,Oh7] for its complete proof).

Proposition 7.6.. Let $\mathcal{U}$ be the Darboux neighborhood of $\Delta$ in $M \times M$ chosen as before. Then, for any given $\alpha>0$ and for any fixed time-independent $J_{0}$, there exists a constant $\delta>0$ such that $\left\|\epsilon G^{\epsilon}\right\|_{C^{2}}<\delta$ and $\left|J-J_{0}\right|_{M \times[0,1]}<\delta$, we have

$$
\int\left|\frac{\partial u}{\partial \tau}\right|_{J}^{2}<A\left(J_{0}, \omega: M\right)-\alpha .
$$

In particular, such a path has trivial homotopy class and so

$$
\int\left|\frac{\partial u}{\partial \tau}\right|_{J}^{2}<\left\|\epsilon G^{\epsilon}\right\|
$$


Moreover, all the other $u \in \mathcal{M}(J, G)$ which are not contained in $\mathcal{M}(J, G: \mathcal{U})$ satisfy

$$
\int\left|\frac{\partial u}{\partial \tau}\right|_{J}^{2}>A\left(J_{0}, \omega: M\right)-\epsilon_{1}
$$

for sufficiently small $\epsilon_{1}=\epsilon_{1}(\delta)$ which is independent of $\alpha$.

By the argument similar to [ $\S 8$, Oh1], we deduce that for $\left(J, \epsilon G^{\epsilon}\right)$ chosen as above, the boundary map

$$
\partial=\partial_{J, \epsilon G^{\epsilon}}: \widetilde{C F}\left(\epsilon G^{\epsilon}\right) \rightarrow \widetilde{C F}\left(\epsilon G^{\epsilon}\right)
$$

is decomposed into

$$
\partial=\partial_{0, \epsilon G^{\epsilon}}+\partial_{\epsilon G^{\epsilon}}^{\prime}
$$

such that $\partial_{\epsilon G^{\epsilon}}^{\prime}$ maps $\widetilde{C F}^{(-\infty, \lambda]}(G) \rightarrow \widetilde{C F}^{\left(-\infty, \lambda-A+\left\|\epsilon G^{\epsilon}\right\|\right]}$. Here the part $\partial_{0}$ is derived from the 'thin' trajectories $u$ and $\partial_{\epsilon G^{\epsilon}}^{\prime}$ from 'thick' trajectories (or from the quantum contributions). In this $C^{2}$-small case where the only time-one periodic orbits are the constant ones, this 'thin' and 'thick' decomposition coincides with that of homotopically trivial and nontrivial trajectories. The essential point of imposing the Hypothesis is that under the Hypothesis, $\partial^{\prime}=0$ and so

$$
\partial=\partial_{0}
$$

This is easy to prove for the weakly exact case (see e.g., [ $\S 3$, Oh1] for the proof in the context of Lagrangian submanifolds). For the proof of this fact for the autonomous Hamiltonians on arbitrary $(M, \omega)$, we refer to the proof of Proposition 7.11 below (or see $[\mathrm{FOn}],[\mathrm{LT}])$.

Now for each given $\epsilon \in\left(0, \epsilon_{1}\right]$, we define the chain map

$$
h_{\epsilon}^{l o c}:\left(\widetilde{C F}(\epsilon k), \partial_{0, \epsilon k}\right) \rightarrow\left(C F\left(\epsilon_{0} G^{\epsilon_{0}}\right), \partial_{0, \epsilon_{0} G^{\epsilon_{0}}}\right)
$$

along the linear path

$$
\mathcal{K}: s \mapsto(1-s) k+s \epsilon_{0} G^{\epsilon_{0}}:=K^{s}
$$

by considering the equation

$$
\left\{\begin{array}{l}
\frac{\partial u}{\partial \tau}+J\left(\frac{\partial u}{\partial t}-X_{K^{\rho(\tau)}}(u)\right)=0 \\
u(-\infty)=p^{-}, u(\infty)=p^{+} \\
w_{\epsilon}^{-} \# u \sim w^{+}
\end{array}\right.
$$

for given $\left[p_{\epsilon}^{-}, w_{\epsilon}^{-}\right] \in$ Crit $\mathcal{A}_{\epsilon k}$ and $\left[p^{+}, w^{+}\right] \in$ Crit $\mathcal{A}_{\epsilon_{0} G^{\epsilon_{0}}}$. The induced homomorphisms

$$
h_{\mathcal{K}}: \widetilde{C F}(J, \epsilon k) \rightarrow \widetilde{C F}\left(J, \epsilon_{0} G^{\epsilon_{0}}\right)
$$

and its local version

$$
h_{\mathcal{K}}^{\text {loc }}: C F(J, \epsilon k ; \mathcal{U}) \rightarrow C F\left(J, \epsilon_{0} G^{\epsilon_{0}} ; \mathcal{U}\right)
$$

induces an isomorphism in the homology with its inverse induced by $h_{\mathcal{K}^{-1}}$ and $h_{\mathcal{K}^{-1}}^{\text {loc }}$ respectively.

Now we consider the Novikov cycles

$$
\beta=\sum a_{[p, w]}[p, w], \quad a_{[p, w]} \in \mathbb{Q} .
$$


The following definition which will be crucial for the minimax argument we carry out later.

Definition 7.7. Let $\beta$ be a Novikov cycle in $\widetilde{C F}(H)$. We define the level of the cycle $\beta$ and denote by

$$
\lambda_{H}(\beta)=\max _{[p, w]}\left\{\mathcal{A}_{H}([p, w]) \mid a_{[p, w]} \neq 0 \text { in }(7.12)\right\}
$$

if $\beta \neq 0$, and just put $\lambda_{H}(0)=+\infty$ as usual.

As in (7.7), we can choose a cycle $\alpha$ for $\left.\left(\widetilde{C F}\left(\epsilon_{0} G^{\epsilon_{0}}\right), \partial_{\left(J, \epsilon_{0} G^{\epsilon_{0}}\right.}\right)\right)$

$$
\alpha=\left[x^{-}, w_{x^{-}}\right]+\sum_{j} a_{j}\left[x_{j}, w_{x_{j}}\right]
$$

with

$$
\mathcal{A}_{\epsilon_{0} G^{\epsilon_{0}}}\left(\left[x_{j}, w_{x_{j}}\right]\right)<\mathcal{A}_{\epsilon_{0} G^{\epsilon_{0}}}\left(\left[x^{-}, w_{x^{-}}\right]\right)
$$

for all $j$, its Floer homology class satisfying $[\alpha] \neq 0$. By considering the local Floer complexes $C F(J, \epsilon k ; \mathcal{U})$ and $C F\left(J, \epsilon_{0} G^{\epsilon_{0}} ; \mathcal{U}\right)$ and their continuation and using the homological essentialness of the maximum point $x^{-}$of $-\epsilon_{0} G^{\epsilon_{0}}$, we can write

$$
\alpha-h_{\mathcal{K}}\left(\alpha_{\epsilon k}\right)=\partial_{\epsilon_{0} G^{\epsilon_{0}}}(\gamma)
$$

for some $\gamma \in C F\left(\epsilon_{0} g^{\epsilon_{0}} ; \mathcal{U}\right)$ for each given $0<\epsilon<\epsilon_{1}$ so that $\alpha_{\epsilon k}$ is a finite union

$$
\alpha_{\epsilon k}=\sum_{i}^{\ell} a_{\left[p_{i}, w_{p_{i}}\right]}\left[p_{i}, w_{p_{i}}\right]
$$

where $p_{i}$ 's are critical points of $k$.

Lemma 7.8. Assume the conditions in Theorem 7.2. Let $\alpha$ be as above. Then for any Novikov cycle $\beta$ homologous to $\alpha$, i.e., satisfying

$$
\alpha=\beta+\partial_{\epsilon_{0} G \epsilon_{0}} \gamma
$$

for some Novikov chain $\gamma \in \widetilde{C F}\left(\epsilon_{0} G^{\epsilon_{0}}\right)$, we have

$$
\lambda_{\epsilon_{0} G^{\epsilon_{0}}}(\beta) \geq \lambda_{\epsilon_{0} G^{\epsilon_{0}}}(\alpha) .
$$

Proof. Note that under the main Hypothesis, we have

$$
\partial_{\epsilon_{0} G^{\epsilon_{0}}}=\partial_{0, \epsilon_{0} G^{\epsilon_{0}}}
$$

for sufficiently small $\epsilon_{0}$. In other words, all the contributions to the boundary $\partial_{\epsilon_{0} G^{\epsilon_{0}}}$ come from 'thin' trajectories. Since $x^{-}$is the maximum point of $-G(\cdot, t)$, there cannot be any such thin trajectory landing at $\left[x^{-}, w_{x^{-}}\right]$.

Therefore $\beta$ must have contribution from $\left[x^{-}, w_{x^{-}}\right]$by (7.14) since $\alpha$ does have contribution from $\left[x^{-}, w_{x^{-}}\right]$. Hence we must have (7.15) by the definition of the level function $\lambda_{\epsilon_{0} G^{\epsilon_{0}}}$. This finishes the proof of the lemma.

Step II: from $\epsilon_{0} G^{\epsilon_{0}}$ to $G$

In this step we consider the homotopy

$$
\mathcal{G}: \eta \mapsto \eta G^{\eta}, \quad \eta \in\left[\epsilon_{0}, 1\right] .
$$


We perturb this to a generic path

$$
\mathcal{H}: \eta \mapsto H(\eta), \eta \in\left[\epsilon_{0}, 1\right], \quad H(1)=G
$$

so that it satisfies the genericity condition as in the Handle sliding lemma (See the paragraph above (6.4)). By the gap condition and the non over-twisting condition in (ii) in Theorem 7.2, we can continue the fixed extremum points $x^{ \pm}$to isolated fixed extremum points of the perturbed path $\mathcal{H}: \eta \mapsto H(\eta)$ without having small periodic points bifurcated from them. In particular the perturbed path $\mathcal{H}$ itself becomes quasiautonomous. Without loss of generality, we may assume that these fixed extrema are the same points $x^{ \pm}$.

Other contractible periodic orbits of $H(\eta)$ will be bifurcated from the constant periodic orbits of $\eta G^{\eta}$. More precisely, we have the following lemma.

Lemma 7.9. For any given $\varepsilon>0$, there exists a generic path $\mathcal{H}: \eta \mapsto H(\eta), \eta \in$ $\left[\epsilon_{0}, 1\right]$ in the above sense such that for each $\eta \in\left[\eta_{0}, 1\right]$, for any contractible periodic orbit $z$ of $H(\eta)$ of period one there exists $x \in$ Crit $_{0}^{\eta} G$ such that

$$
\|z-x\|_{C^{2}}<\varepsilon
$$

(2) there exists a canonical small cylinder $v$ (up to homotopy) connecting $z$ and $x$ such that

$$
\left|\mathcal{A}_{H(\eta)}\left(\left[z, w_{z}\right]\right)-\mathcal{A}_{\eta G^{\eta}}\left(\left[x, w_{x}\right]\right)\right|<\varepsilon
$$

and

$$
\mathcal{A}_{H(\eta)}\left(\left[x^{-}, w_{x^{-}}\right]\right)-\mathcal{A}_{H(\eta)}\left(\left[z, w_{z}\right]\right)>\frac{1}{2} \epsilon_{0} \delta_{1}
$$

where $w_{z} \sim w_{x} \# v$.

The point of Remark 7.3 (1) is that the length minimizing property holds for the Hamiltonian path $\eta \mapsto H(\eta)$ which is perturbed from $G$ and this Hamiltonian satisfies the property assumed in Theorem I (i). Indeed the proof below proves that this path is length minimizing. Using Lemma 5.1, we then derive the length minimizing property of $G$ itself.

As in $\S 3$, we consider the partition

$$
I: \eta_{0}=\epsilon_{0}<\eta_{1}<\eta_{2}<\cdots<\eta_{N}=1
$$

and denote its mesh of $I$ by

$$
\Delta_{I}=\max _{j}\left|\eta_{j+1}-\eta_{j}\right|
$$

We also consider the associated piecewise linear homotopy

$$
\mathcal{H}_{I}:=\mathcal{L}_{1} \# \mathcal{L}_{2} \# \cdots \# \mathcal{L}_{N}
$$

where $\mathcal{L}_{j}$ is the linear homotopy

$$
s \mapsto(1-s) H\left(\eta_{j-1}\right)+s H\left(\eta_{j}\right) .
$$

We call the above piecewise linear homotopy $\mathcal{H}_{I}$ the adiabatic homotopy associated to $\mathcal{H}$ and the partition $I$. We also denote the associated chain map

$$
h_{\mathcal{G}_{I}}:=h_{\eta_{N} \eta_{N-1}}^{\mathcal{G}, l i n} \circ \cdots \circ h_{\eta_{1} \epsilon_{0}}^{\mathcal{G}, l i n}: \widetilde{C F}\left(H^{\epsilon_{0}}\right) \rightarrow \widetilde{C F}(H(1))
$$


the adiabatic chain map associated to $\mathcal{H}$ and $I$. We will just denote $\mathcal{H}^{\text {adb }}$ and $h_{\mathcal{H}}^{\text {adb }}$ respectively for the adiabatic homotopy and the adiabatic chain map associated to $\mathcal{H}$ when we do not need to specify the partition $I$.

Now we choose $I$ with $\Delta_{I}$ so small that

$$
\begin{aligned}
\Delta\left(\mathcal{H}_{I}\right), \Delta\left(\mathcal{H}_{I}^{-1}\right), \Delta_{I} \cdot\|H\|_{C^{0}} & <\frac{1}{6} \epsilon_{0} \delta_{1} \\
\int_{0}^{1}\left|H\left(\eta_{j+1}\right)\left(x^{-}, t\right)-H\left(\eta_{j}\right)\left(x^{-}, t\right)\right| d t & <\frac{1}{6} \epsilon_{0} \delta_{1} .
\end{aligned}
$$

We recall the Handle sliding lemma, Proposition 6.3, applied to our perturbed family $\mathcal{H}$. It is easy to see from the definition that we have

$$
A_{(j, \mathcal{H})}^{0} \geq \frac{3}{4} A_{(j, \mathcal{G})}
$$

if $\mathcal{H}$ is sufficiently $C^{\infty}$-close to $\mathcal{G}$, where the constants $A_{(j, \mathcal{H})}^{0}, A_{(j, \mathcal{G})}$ are defined as in (6.3) and (6.5). Because there does not occur bifurcation of contractible periodic orbits along the family $\mathcal{G}$, a Gromov compactness type argument proves $A_{(j, \mathcal{G})}>0$. We now state a version of the Handle sliding lemma that we need in our proof.

Proposition 7.10. Let $\mathcal{G}$ and $\mathcal{H}$ be as above and $j=\left\{J^{\eta}\right\}$ be a smooth periodic (two parameter) family of compatible almost complex structures. Let $\eta<\eta^{\prime} \in[0,1]$. Then for any fixed $j$ and for any $\varepsilon>0$, there exists a constant $\delta>0$ such that if $0 \leq \eta^{\prime}-\eta<\delta$, any finite energy solution of

$$
\frac{\partial u}{\partial \tau}+J^{\rho(\tau)}\left(\frac{\partial u}{\partial t}-X_{H^{\rho(\tau)}}(u)\right)=0
$$

must be either satisfies

$$
\int\left|\frac{\partial u}{\partial \tau}\right|^{2} \leq \varepsilon
$$

or

$$
\int\left|\frac{\partial u}{\partial \tau}\right|^{2} \geq \frac{1}{2} A_{(j, \mathcal{G})}
$$

Here $H^{s}$ is the linear path $H^{s}=(1-s) H\left(\eta_{1}\right)+s H\left(\eta_{2}\right)$ and $\rho$ is the standard function as before.

By choosing $\delta_{1}$ and then $\Delta_{I}$ sufficiently small, we will also make the constant $A_{(j, \mathcal{G})}$, satisfy

$$
A_{(j, \mathcal{G})}>3 \delta_{1}
$$

which is possible because $A_{(j, \mathcal{G})}$ depends only on $\epsilon_{0}$ and $\mathcal{G}$ but independent of $\delta_{1}$.

Next we consider the cycle

$$
\alpha_{G}=\alpha_{H(1)}:=h_{\mathcal{H}}^{a d b}(\alpha)
$$

and prove the following proposition, where the condition of no quantum contribution enters.

Proposition 7.11. (Non-PUSHING DOWN LEMma II) Let $G$ and $(M, \omega)$ as in Theorem 7.2. Then the cycle $\alpha_{H(1)}$ has the properties

(1) $\lambda_{H(1)}\left(\alpha_{H(1)}\right)=-\int_{0}^{1} H(1)\left(x^{-}, t\right) d t$ 
(2) Non pushing-down lemma for $\alpha_{H(1)}$ holds, i.e., for any Novikov cycle $\beta \in \widetilde{C F}(H(1))$ homologous to $\alpha_{H(1)}$, we have

$$
\lambda_{H(1)}(\beta) \geq \lambda_{H(1)}\left(\alpha_{H(1)}\right)
$$

Proof. We consider the family of cycles

$$
\alpha_{j}=h_{\eta_{j} \eta_{j+1}}^{\mathcal{H}, \text { lin }} \circ \cdots \circ h_{\eta_{1} \epsilon}^{\mathcal{H}, \text { lin }}(\alpha) \in \widetilde{C F}\left(H\left(\epsilon_{j+1}\right)\right)
$$

for $j=0, \cdots, N-1$. We will prove the following properties of the cycle $\alpha_{j}$ inductively over $j$ :

(P1.j) $\alpha_{j}$ gets non-trivial contribution from $\left[x^{-}, w_{x^{-}}\right] \in$ Crit $\mathcal{A}_{H(\eta)}$,

(P2.j) its level satisfies

$$
\lambda_{H\left(\eta_{j}\right)}\left(\alpha_{j}\right)=-\int_{0}^{\eta_{j}} H\left(\eta_{j}\right)\left(x^{-}, t\right) d t
$$

(P3.j) Non pushing down lemma for $\alpha_{j}$ holds, i.e., for any Novikov cycle $\beta_{j}$ homologous to $\alpha_{j}$, we have

$$
\lambda_{H\left(\eta_{j}\right)}\left(\beta_{j}\right) \geq \lambda_{H\left(\eta_{j}\right)}\left(\alpha_{j}\right)
$$

Once we prove this, Proposition 7.11 will follow by putting $j=N-1$.

For $j=0,(\mathrm{P} 1),(\mathrm{P} 2)$ follow from the definition of $\alpha$ and (P3) follows from Lemma 7.8. Now suppose (P1-3.j) hold for $j$ and we will prove $(\mathrm{P} 1-3 . \mathrm{j}+1)$. We first prove $(P 1 . j+1)$ and $(P 2 . j+1)$. We note that

$$
h_{\eta_{j+1} \eta_{j}}^{\mathcal{H}^{-1}, \operatorname{lin}} \circ h_{\eta_{j} \eta_{j+1}}^{\mathcal{H}, \text { lin }}\left(\alpha_{j}\right)
$$

is homologous to $\alpha_{j}$ and so by (P3.j), we have

$$
\lambda_{H\left(\eta_{j}\right)}\left(h_{\eta_{j+1} \eta_{j}}^{\mathcal{H}^{-1}, \text { lin }} \circ h_{\eta_{j} \eta_{j+1}}^{\mathcal{H}, \text { lin }}\left(\alpha_{j}\right)\right) \geq \lambda_{H\left(\eta_{j}\right)}\left(\alpha_{j}\right)=-\int_{0}^{\eta_{j}} H\left(\eta_{j}\right)\left(x^{-}, t\right) d t .
$$

Therefore (7.19) and (P2.j) together with the upper estimate imply

$$
\lambda_{H\left(\eta_{j}\right)}\left(h_{\eta_{j+1} \eta_{j}}^{\mathcal{H}^{-1}, \text { lin }} \circ h_{\eta_{j} \eta_{j+1}}^{\mathcal{H}, l i n}\left(\alpha_{j}\right)\right)-\lambda_{H\left(\eta_{j+1}\right)}\left(h_{\eta_{j} \eta_{j+1}}^{\mathcal{H}, l i n}\left(\alpha_{j}\right)\right) \leq \frac{1}{6} \epsilon_{0} \delta_{1}
$$

and so

$$
\lambda_{H\left(\eta_{j+1}\right)}\left(h_{\eta_{j} \eta_{j+1}}^{\mathcal{H}, l i n}\left(\alpha_{j}\right)\right) \geq-\int_{0}^{\eta_{j}} H\left(\eta_{j}\right)\left(x^{-}, t\right) d t-\frac{1}{6} \epsilon_{0} \delta_{1}
$$

This together with Proposition 7.10 and by (7.4), also implies that any trajectory starting from the cycle $\alpha_{j}$ that lands at the critical point and realizes the level $\lambda_{H\left(\eta_{j+1}\right)}\left(\alpha_{H \eta_{j+1}}\right)$ must be very short: for not very short path $u$ staring from $[z, w] \neq$ $\left[x^{-}, w_{x^{-}}\right]$a generator of $\alpha_{j}$, it follows from (7.24)

$$
\mathcal{A}_{H\left(\eta_{j+1}\right)}(u(\infty))-\mathcal{A}_{H\left(\eta_{j}\right)}(u(-\infty)) \leq-\frac{1}{2} A_{(j, \mathcal{G})} \leq-\frac{3}{2} \delta_{1}
$$


and so

$$
\begin{aligned}
\mathcal{A}_{H\left(\eta_{j+1}\right)}(u(\infty)) & \leq \mathcal{A}_{H\left(\eta_{j}\right)}(u(-\infty))-\frac{3}{2} \delta_{1} \\
& \leq \mathcal{A}_{H\left(\eta_{j}\right)}\left(\left[x^{-}, w_{x^{-}}\right]\right)+\frac{1}{2} \epsilon_{0} \delta_{1}-\frac{3}{2} \delta_{1} \\
& \leq-\int_{0}^{\eta_{j}} H\left(\eta_{j}\right)\left(x^{-}, t\right) d t+\frac{1}{2} \epsilon_{0} \delta_{1}-\frac{3}{2} \delta_{1}
\end{aligned}
$$

Here the last inequality follows from (7.19), (7.20) and (P2.j). Therefore it follows from (7.28) that such trajectory $u$ cannot land at a critical point that realizes the level of $\alpha_{j+1}$ since

$$
\frac{3}{2} \delta_{1}-\frac{1}{2} \epsilon_{0} \delta_{1}>\frac{1}{6} \epsilon_{0} \delta_{1}
$$

Because of (7.18) and the upper estimate, it follows that any generator $[z, w]$ with $[z, w] \neq\left[x^{-}, w_{x^{-}}\right]$cannot land at the critical point of $\mathcal{A}_{H\left(\eta_{j+1}\right)}$ that realizes the level of $\alpha_{j+1}$. This proves that the only possible path realizing the level of $\alpha_{j}$ is a very short path $u$ such that

$$
u(-\infty)=\left[x^{-}, w_{x^{-}}\right], u(\infty)=\left[x^{-}, w_{x^{-}}\right]
$$

This prove $(P 1 . j+1)$ and $(P 2 . j+1)$.

Now it remains to prove $(\mathrm{P} 3 . \mathrm{j}+1)$. We prove this by contradiction. Suppose that there is a Novikov cycle $\beta_{j+1} \in \widehat{C F}\left(H\left(\eta_{j+1}\right)\right)$ homologous to $\alpha_{j+1}$, i.e.,

$$
\alpha_{j+1}=\beta_{j+1}+\partial \gamma_{j+1}
$$

but

$$
\lambda_{\eta_{j+1} G^{\eta_{j+1}}}\left(\beta_{j+1}\right)<\lambda_{\eta_{j+1} G^{\eta_{j+1}}}\left(\alpha_{j+1}\right) .
$$

We study the two cases separately:

(1) where $(M, \omega)$ is weakly exact

(2) where $G$ is autonomous.

In the case where $(M, \omega)$ is weakly exact, (7.31) indeed implies

$$
\lambda_{H\left(\eta_{j+1}\right)}\left(\beta_{j+1}\right)<\lambda_{H\left(\eta_{j+1}\right)}\left(\alpha_{j+1}\right)-\frac{1}{2} \epsilon_{0} \delta_{1}
$$

by (7.18) because the action depends only on $z$ not on the choice of $w$. Then the 
upper estimate, (7.19) and (7.20) imply

$$
\begin{aligned}
\lambda_{H\left(\eta_{j}\right)}\left(h_{\eta_{j+1} \mathcal{H}_{j}^{-1}, l i n}^{\mathcal{H}_{j}}\left(\beta_{j+1}\right)\right) \leq & \lambda_{H\left(\eta_{j+1}\right)}\left(\beta_{j+1}\right)+\frac{1}{6} \epsilon_{0} \delta_{1} \\
< & \lambda_{H\left(\eta_{j+1}\right)}\left(\alpha_{j+1}\right)-\frac{1}{2} \epsilon_{0} \delta_{1}+\frac{1}{6} \epsilon_{0} \delta_{1} \\
= & -\int_{0}^{\eta_{j+1}} H\left(\eta_{j+1}\right)\left(x^{-}, t\right) d t-\frac{1}{3} \epsilon_{0} \delta_{1} \\
\leq & -\int_{0}^{\eta_{j}} H\left(\eta_{j}\right)\left(x^{-}, t\right) d t-\int_{\eta_{j}}^{\eta_{j+1}} H\left(\eta_{j+1}\right)\left(x^{-}, t\right) d t \\
& -\int_{0}^{\eta_{j}}\left(H\left(\eta_{j+1}\right)\left(x^{-}, t\right)-H\left(\eta_{j}\right)\left(x^{-}, t\right)\right) d t-\frac{1}{3} \epsilon_{0} \delta_{1} \\
\leq & -\int_{0}^{\eta_{j}} H\left(\eta_{j}\right)\left(x^{-}, t\right) d t+\frac{1}{3} \epsilon_{0} \delta_{1}-\frac{1}{3} \epsilon_{0} \delta_{1} \\
= & -\int_{0}^{\eta_{j}} H\left(\eta_{j}\right)\left(x^{-}, t\right) d t=\lambda_{H\left(\eta_{j}\right)}\left(\alpha_{j}\right)
\end{aligned}
$$

and hence

$$
\lambda_{H\left(\eta_{j}\right)}\left(h_{\eta_{j+1} \eta_{j}}^{\mathcal{H}^{-1}, \operatorname{lin}}\left(\beta_{j+1}\right)\right)<\lambda_{H\left(\eta_{j}\right)}\left(\alpha_{j}\right) .
$$

However (7.33) is a contradiction to (P3.j) since the cycle $h_{\eta_{j+1} \mathcal{G}^{-1}, l i n}\left(\beta_{j+1}\right)$ is homologous to

$$
h_{\eta_{j+1} \eta_{j}}^{\mathcal{G}^{-1}, l i n}\left(\alpha_{j+1}\right)=h_{\eta_{j+1} \eta_{j}}^{\mathcal{G}^{-1}, l i n} \circ h_{\eta_{j} \eta_{j+1}}^{\mathcal{G}, l i n}\left(\alpha_{j}\right)
$$

which is in turn homologous to $\alpha_{j}$. This finishes the proof of $(\mathrm{P} 3 . \mathrm{j}+1)$ for this case (1).

When $G$ is autonomous, we use a generic family of $\mathcal{H}=\{H(\eta)\}$ of autonomous Hamiltonians $H(\eta)$ which are Morse except at a finite set of $\eta$ 's, and of $j=\left\{J^{\eta}\right\}$ where each $J^{\eta}$ is $t$-independent. Since $x^{-}$is the minimum point of $H(\eta)$, there is no $t$ independent trajectory of $\mathcal{A}_{H(\eta)}$ landing at $\left[x^{-}, w_{x^{-}}\right]$. Therefore any Floer trajectory landing at $\left[x^{-}, w_{x^{-}}\right]$must be $t$-dependent. Let the trajectory start at $[x, w], x \in$ Crit $H(\eta)$ with

$$
\mu([x, w])-\mu\left(\left[x^{-}, w_{x^{-}}\right]\right)=1
$$

and denote by $\mathcal{M}_{\left(J^{\eta}, H(\eta)\right)}\left([x, w],\left[x^{-}, w_{x^{-}}\right]\right)$the corresponding Floer moduli space of connecting trajectories. The general index formula shows

$$
\mu([x, w])=\mu\left(\left[x, w_{x}\right]\right)-2 c_{1}([w]) .
$$

We consider two cases separately: the cases of $c_{1}([w])=0$ or $c_{1}([w]) \neq 0$. If $c_{1}([w]) \neq$ 0 , we derive from (7.34), (7.35) that $x \neq x^{-}$. This implies that any such trajectory must come with (locally) free $S^{1}$-action, i.e., the moduli space

$$
\widehat{\mathcal{M}}_{\left(J^{\eta}, H(\eta)\right)}\left([x, w],\left[x^{-}, w_{x^{-}}\right]\right)=\mathcal{M}_{\left(J^{\eta}, H(\eta)\right)}\left([x, w],\left[x^{-}, w_{x^{-}}\right]\right) / \mathbb{R}
$$

and its stable map compactification have a locally free $S^{1}$-action without fixed points. Therefore after a $S^{1}$-invariant perturbation $\Xi$ via considering the quotient Kuranishi structure [FOn] on the quotient space $\widehat{\mathcal{M}}_{\left(J^{\eta}, H(\eta)\right)}\left([x, w],\left[x^{-}, w_{x^{-}}\right]\right) / S^{1}$, the corresponding perturbed moduli space $\widehat{\mathcal{M}}_{\left(J^{\eta}, H(\eta)\right)}\left([x, w],\left[x^{-}, w_{x^{-}}\right] ; \Xi\right)$ becomes empty. This is because the quotient Kuranishi structure has the virtual dimension -1 by the 
assumption (7.34). We refer to [FOn] or [LT] for more explanation on this $S^{1}$-invariant regularization process. Now consider the case $c_{1}([w])=0$. First note that (7.34) and (7.35) imply that $x \neq x^{-}$. On the other hand, if $x \neq x^{-}$, the same argument as above shows that the perturbed moduli space becomes empty.

It now follows that there is no trajectory of index 1 that land at $\left[x^{-}, w_{x^{-}}\right]$after the $S^{1}$-invariant regularization. This together with (7.31) gives rise to a contradiction to (7.30) as in Lemma 7.8 and finishes the proof of $(\mathrm{P} 3 . \mathrm{j}+1)$ for the second case (2). Hence the proof of Proposition 7.11.

REMARK 7.12. (1) We would like to note that a (Morse) gradient trajectory of the Morse function $H(\eta)$ is not necessarily regular as a Floer gradient trajectory i.e., as a solution of the perturbed Cauchy-Riemann equation, unless the $C^{2}$-norm of $H(\eta)$ is sufficiently small. The "slowness" condition introduced in [En], [MS] is related to this problem.

(2) A careful look of the above proofs shows that the only obstacle to extending them to arbitrary quasi-autonomous Hamiltonians on general symplectic manifolds is that Non pushing-down lemma will not be available for the cycle

$$
\alpha_{G}=\alpha_{H(1)}=h_{\mathcal{H}}^{a d b}(\alpha)
$$

defined in (7.26) in case quantum contribution exists for the Floer boundary operator. This will prevent us from using the deformation argument used in the end of $\S 7$ to produce a solution for the continuity equation along the linear path $\mathcal{L}$. Some simpleness condition as in $[\mathrm{BP}]$ enables us to prove Non-pushing down lemma, which we will investigate further elsewhere.

Step III; from $G$ to $F$

Now we consider the homotopy $\mathcal{F}=\left\{F^{s}\right\}_{0 \leq s \leq 1}$

$$
G \mapsto F \text {. }
$$

We take a partition

$$
I: 0=s_{0}<s_{1}<\cdots<s_{N-1}<s_{N}=1
$$

and its associated adiabatic homotopy $\mathcal{F}^{a d b}$.

We first recall from Proposition 2.3 that

$$
\operatorname{Spec}\left(F^{s}\right)=\operatorname{Spec}(G)
$$

which is a measure zero subset $\mathbb{R}$. We consider the family of cycles

$$
h_{\mathcal{F}^{s}}^{a d b}\left(\alpha_{G}\right), \quad s \in[0,1]
$$

and its level function

$$
\mu(s):=\lambda_{F^{s}}\left(h_{\mathcal{F}^{s}}^{a d b}\left(\alpha_{G}\right)\right), \quad s \in[0,1] .
$$

Here $\mathcal{F}^{s}$ is the path $t \mapsto F^{t s}, t \in[0,1]$. We will provide the proof of the following proposition in the appendix.

Proposition 7.13. The function $\mu$ is continuous and so constant. In particular, the cycle

$$
\alpha_{F}:=h_{\mathcal{F}}^{a d b}\left(\alpha_{G}\right)
$$


has the level

$$
\lambda_{F}\left(\alpha_{F}\right)=\lambda_{G}\left(\alpha_{G}\right)=-\int_{0}^{1} G\left(x^{-}, t\right) d t .
$$

With this proposition at our disposal, we prove

Proposition 7.14 (NON PUSHING-DOWN LEMMA III). Let $\alpha_{F}$ be as above. If a Novikov cycle $\beta^{\prime}$ is homologous to $\alpha_{F}$ in $\widetilde{C F}(F)$, i.e., satisfies

$$
\alpha_{F}=\beta^{\prime}+\partial_{F}\left(\gamma^{\prime}\right)
$$

then we must have

$$
\lambda_{F}\left(\beta^{\prime}\right) \geq \lambda_{F}\left(\alpha_{F}\right)
$$

Proof. Suppose the contrary that there exists $\beta^{\prime}$ and $\gamma^{\prime}$ with (7.37) and

$$
\lambda_{F}\left(\beta^{\prime}\right)<\lambda_{F}\left(\alpha_{F}\right)
$$

satisfied. We apply the homotopy $h_{\mathcal{F}^{-1}}^{a d b}$ to (7.37). Composing this with $h_{\mathcal{F}}^{a d b}$, we get the identity

$$
i d-h_{\mathcal{F}-1}^{a d b} \circ h_{\mathcal{F}}^{a d b}=\partial_{F} \circ \widetilde{H}+\widetilde{H} \circ \partial_{G}
$$

for the obvious Floer chain homotopy $\widetilde{H}: \widetilde{C F}(G) \rightarrow \widetilde{C F}(G)$ in a standard way. We apply (7.40) to the cycle $\alpha_{G}$ to get

$$
\alpha_{G}-h_{\mathcal{F}-1}^{a d b}\left(\alpha_{F}\right)=\partial_{G} \tilde{H}\left(\alpha_{G}\right)
$$

from the definition of $\alpha_{F}$ in (7.41). Inserting (7.41) into (7.40) and using the chain property of $h_{\mathcal{F}-1}^{a d b}$, we get

$$
\alpha_{G}-h_{\mathcal{F}-1}^{a d b}\left(\beta^{\prime}\right)=\partial_{G}\left(\widetilde{H}\left(\alpha_{G}\right)+h_{\mathcal{F}-1}^{a d b}\left(\gamma^{\prime}\right)\right)
$$

Lemma 7.8 implies that

$$
\lambda_{G}\left(h_{\mathcal{F}-1}^{a d b}\left(\beta^{\prime}\right)\right) \geq \lambda_{G}\left(\alpha_{G}\right)=c^{+}
$$

On the other hand, using (7.39), (7.43) and the Handle sliding lemma, and applying the proof of Proposition 7.13 in Appendix to $\beta^{\prime}$ backwards, $F \mapsto G$, we prove that the function $s \mapsto \lambda_{F F^{s}}^{\mathcal{F}^{-1}, a d b}\left(\beta^{\prime}\right)$ is continuous and so constant. In particular, we have

$$
\lambda_{G}\left(h_{\mathcal{F}^{-1}}^{a d b}\left(\beta^{\prime}\right)\right)=\lambda_{F}\left(\beta^{\prime}\right) .
$$

Therefore we have proven

$$
\lambda_{G}\left(h_{\mathcal{F}^{-1}}^{a d b}\left(\beta^{\prime}\right)\right)=\lambda_{F}\left(\beta^{\prime}\right)<\lambda_{F}\left(\alpha_{F}\right)=\lambda_{G}\left(\alpha_{G}\right)
$$

Now (7.43) and (7.44) give rise to a contradiction. This finishes the proof.

Step IV: from the $\mathcal{K} \#_{R_{1}} \mathcal{G}^{a d b} \#_{R_{2}} \mathcal{F}^{a d b}$ to $\mathcal{L}$

Finally we consider the linear homotopy $\mathcal{L}=\left\{L^{s}\right\}_{0 \leq s \leq 1}$ from $\epsilon k$ to $F$

$$
L^{s}=(1-s) \epsilon k+s F
$$

and the associated chain map

$$
h_{\mathcal{L}}: \widetilde{C F}\left(J^{0}, \epsilon k\right) \rightarrow \widetilde{C F}\left(J^{1}, F\right)
$$

(by connecting $J^{0}$ and $J^{1}$ by a generic path $\left\{J^{s}\right\}$ ). 
We connect the glued homotopy $\mathcal{L}_{0}=\mathcal{K} \#_{R_{1}} \mathcal{G}^{a d b} \#_{R_{2}} \# \mathcal{F}^{a d b}$ and $\mathcal{L}_{1}=\mathcal{L}$ by any generic homotopy (of homotopies) $\overline{\mathcal{L}}=\left\{\mathcal{L}_{\kappa}\right\}_{0 \leq \kappa \leq 1}$ and consider the parameterized equation

$$
\frac{\partial u}{\partial \tau}+J^{\rho_{1}(\tau)}\left(\frac{\partial u}{\partial t}-X_{H_{\kappa}^{\rho_{2}(\tau)}}(u)\right)=0
$$

for $\kappa \in[0,1]$. Again this parameterized equation induces the identity

$$
h_{\mathcal{L}}-h_{\mathcal{K}_{R_{1}} \mathcal{G}^{a d b} \#_{R_{2}} \mathcal{F}^{a d b}}=H_{\overline{\mathcal{L}}} \partial_{\left(J^{0}, \epsilon k\right)}+\partial_{\left(J^{1}, F\right)} H_{\overline{\mathcal{L}}}
$$

for the corresponding chain homotopy $H_{\overline{\mathcal{L}}}: \widetilde{C F}\left(J^{0}, \epsilon k\right) \rightarrow \widetilde{C F}\left(J^{1}, F\right)$. Applying this identity to $\alpha_{\epsilon k}$ above, we have

$$
h_{\mathcal{L}}\left(\alpha_{\epsilon k}\right)-h_{\mathcal{K}_{R_{1}} \mathcal{G} \#_{R_{2}} \mathcal{F}^{a d b}}\left(\alpha_{\epsilon k}\right)=\partial_{\left(J^{1}, F\right)} H_{\overline{\mathcal{L}}}\left(\alpha_{\epsilon k}\right) .
$$

Since the standard gluing theorem in the Floer theory implies

$$
h_{\mathcal{K}_{R_{1}} \mathcal{G}^{a d b} \#_{R_{2}} \mathcal{F}^{a d b}}=h_{\mathcal{F}}^{a d b} \circ h_{\mathcal{G}}^{a d b} \circ h_{\mathcal{K}}
$$

for sufficiently large $R_{i}>0$, we have

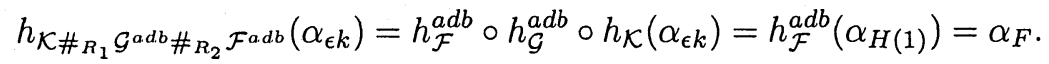

Obviously $h_{\mathcal{L}}\left(\alpha_{\epsilon k}\right)$ is a Novikov cycle in $\widetilde{C F}\left(J^{1}, F\right)$. Therefore Proposition 7.14 implies that

$$
\lambda_{F}\left(h_{\mathcal{L}}\left(\alpha_{\epsilon k}\right)\right) \geq \lambda_{F}\left(\alpha_{F}\right) .
$$

By the definition of the chain map $h_{\mathcal{L}}$ and the cycle $\alpha_{\epsilon k}$ in (7.13), this then implies existence of $\left[y, w_{y}\right] \in \widetilde{C F}\left(J^{0}, \epsilon k\right)$ and $[z, w] \in \widetilde{C F}\left(J^{1}, F\right)$ for which there exists a solution of the following Cauchy-Riemann equation:

$$
\left\{\begin{array}{l}
\frac{\partial u}{\partial \tau}+J^{\rho_{1}(\tau)}\left(\frac{\partial u}{\partial t}-X_{L^{\rho_{2}(\tau)}}(u)\right)=0 \\
u(-\infty)=y, \quad u(\infty)=z \\
w_{y} \# u \sim w
\end{array}\right.
$$

with

$$
\mathcal{A}_{F}([z, w]) \geq \lambda_{F}\left(\alpha_{F}\right)=\mathcal{A}_{F}\left(\left[z^{-}, w_{z^{-}}\right]\right) .
$$

This is exactly what we wanted to prove in Proposition 5.3. This finally finishes the proof of Proposition 5.3 and hence the proof of Theorem I.

8. Construction of spectral invariants. In this section, we outline our construction of the spectral invariants of the Viterbo type [V] (more precisely, the type the author constructed in $[\mathrm{Oh} 3,5])$ on arbitrary compact symplectic manifolds. As a consequence, we also define a new invariant norm on the Hamiltonian diffeomorphism group of arbitrary compact symplectic manifolds. We just illustrate the main idea of the construction in the present paper with minimal possible sophistication in the presentation and refer readers to [Oh7] for precise details of the construction.

The starting point of our construction of the invariants will then be the fact that for any fixed generic autonomous Hamiltonian $g$ on $M$ we have the isomorphism

$$
\left(C F_{*}\left(\epsilon g ; \Lambda_{\omega}\right), \partial_{\epsilon g}\right) \simeq\left(C M_{*}(-\epsilon g ; \mathbb{Q}), \partial_{-\epsilon g}^{\text {Morse }}\right) \otimes \Lambda_{\omega}
$$


as a chain complex when $\epsilon>0$ is sufficiently small and the canonical isomorphism

$$
h_{\epsilon g H}: H F_{*}\left(\epsilon g ; \Lambda_{\omega}\right) \rightarrow H F_{*}\left(H ; \Lambda_{\omega}\right)
$$

for any Hamiltonian $H$ over the Novikov ring $\Lambda_{\omega}$. The natural isomorphism (8.2) is induced by the chain map

$$
h_{\epsilon g H}: \widetilde{C F}(\epsilon g) \rightarrow \widetilde{C F}(H)
$$

over the linear path $H^{s}=(1-s) \epsilon g+s H$. Here we also note that (8.1) also induces a canonical isomorphism

$$
H F_{*}\left(\epsilon g ; \Lambda_{\omega}\right) \simeq H M_{*}(-\epsilon g ; \mathbb{Q}) \otimes \Lambda_{\omega}
$$

Here $C M_{*}(-\epsilon g ; \mathbb{Q})$ and $H M_{*}(-\epsilon g ; \mathbb{Q})$ denote the Morse chain complex and its associated homology of $-\epsilon g$ with the $\mathbb{Q}$-coefficients.

By letting $\epsilon \rightarrow 0$, we will have the corresponding limit isomorphism

$$
h_{H}: H_{*}(M ; \mathbb{Q}) \otimes \Lambda_{\omega} \rightarrow H F^{*}\left(H ; \Lambda_{\omega}\right)
$$

by identifying the singular cohomology $H^{*}(M, \mathbb{Q})$ with $H M^{*}(\epsilon g ; \mathbb{Q})$ by realizing its Poincaré dual by a Morse cycle of $-\epsilon g$ and then composing with the map (8.1).

Definition 8.1. Let $H$ be a given generic Hamiltonian. For each $a \neq 0 \in$ $H^{*}(M ; \mathbb{Q})$, we denote by $P D(a)$ its Poincaré dual to $a$. We consider the Floer homology class $h_{\epsilon g H}(P D(a)) \in H F^{*}\left(H ; \Lambda_{\omega}\right)$. We define the level of the Floer homology class $h_{\epsilon g H}(P D(a))$ by

$$
\rho(H ; a)=\lim _{\epsilon \rightarrow 0} \inf \left\{\lambda_{H}(\alpha) \mid[\alpha]=h_{\epsilon g H}(P D(a)), \alpha \in \widetilde{C F}(H)\right\} .
$$

Of course, a crucial task in this definition is to show that this is well-defined, i.e, the numbers are finite, independent of the choice of the Morse function $g$ and behave continuously over $H$ (in $C^{0}$-norm). The following theorem is the analog to [Theorem II, Oh5] which can be proved in a similar way. However we exploit the isomorphism (8.1) in a crucial way here.

Theorem 8.2. Let $H$ be a given Hamiltonian. For each $a \neq 0 \in H^{*}(M ; \mathbb{Q})$, the number $\rho(H ; a)$ is finite and the assignment $H \mapsto \rho(H ; a)$ can be extended to $C^{0}(M \times[0,1])$ as a continuous function with respect to $C^{0}$-topology of $H$.

Proof. The proof will be the same as [Oh5] once we prove the finiteness of the value $\rho(H ; a)$.

To be more precise, we choose a Morse function $g$ on $M$ and use the chain map (8.3). The homology class $P D(a)$ considered as a Morse homology class of $-\epsilon g$ defines a Floer homology class of $\epsilon g$ which is non-zero by the fact that the Floer boundary operator $\partial_{\epsilon g} \simeq \partial_{-\epsilon g}^{M \text { orse }} \otimes \Lambda_{\omega}$. Therefore we have

$$
\inf _{[\alpha]=h_{\epsilon g H}(P D(a))} \lambda_{H}(\alpha)<\infty
$$

since $h_{\epsilon g H}(P D(a)) \neq 0$. In fact, by the same calculation as in Proposition 3.2, we can prove

$$
\rho(H ; a) \leq \int_{0}^{1}-\min H d t .
$$


To prove $\rho(H ; a)>-\infty$, we first prove the following lemma.

LEMMA 8.3. We have

$$
\rho(\epsilon g: a) \geq-\max \epsilon g \text {. }
$$

Proof. Let $\gamma \in \widetilde{C F}(\epsilon g)$ be a Novikov cycle with $[\gamma]=P D(a)$. We write

$$
\gamma=\gamma_{0}+\gamma^{\prime}
$$

where $\gamma_{0}$ is the sum of the terms with trivial homotopy class i.e., those of the type with $\left[x, w_{x}\right], x \in$ Crit $g$ and $\gamma^{\prime}$ are the ones $[x, w]$ with non-trivial homotopy class with $[w] \neq 0 \in \Gamma_{\omega}$. Since $\partial_{\epsilon g}$ preserves this decomposition (no quantum contribution!) and since any $b \in H_{*}(M ; \mathbb{Q})$ can be represented by $\gamma_{0}$, both $\gamma_{0}$ and $\gamma^{\prime}$ are closed and satisfy

$$
\left[\gamma_{0}\right]=b \quad \text { and }\left[\gamma^{\prime}\right]=0 \text {. }
$$

By setting $0 \neq b=P D(a)=\left[\gamma_{0}\right]$ in the Morse homology of $\epsilon g$, we have $\gamma_{0} \neq 0$. An easy fact from the (finite dimensional) Morse homology theory implies

$$
\lambda_{\epsilon g}\left(\gamma_{0}\right) \geq \min (-\epsilon g)=-\max (\epsilon g) .
$$

Obviously since we have $\lambda_{\epsilon g}(\gamma) \geq \lambda_{\epsilon g}\left(\gamma_{0}\right)$, (8.9) finishes the proof.

Now we go back to the proof of Theorem 8.2. Let $\alpha \in \widetilde{C F}(H)$ with its Floer homology class $[\alpha]=h_{\epsilon g H}(P D(a))$. Note that by the same calculation as that in Proposition 3.2 along the linear path from $H$ to $\epsilon g$, we have

$$
\lambda_{\epsilon g}\left(h_{H \epsilon g}^{\operatorname{lin}}(\alpha)\right) \leq \lambda_{H}(\alpha)+\int_{0}^{1}-\min (\epsilon g-H) d t
$$

where we know $h_{H \epsilon g}^{\text {lin }}(\alpha) \neq 0$ because $\left[h_{H \epsilon g}^{\text {lin }}(\alpha)\right] \neq 0$ since $[\alpha] \neq 0$ and $h_{H \epsilon g}^{\text {lin }}$ induces an isomorphism in homology. On the other hand, let $\gamma_{0}$ be a representative as in Lemma 8.3 with $\left[\gamma_{0}\right]=b$. Since $[\alpha]=h_{\epsilon g H}^{\operatorname{lin}}\left(\gamma_{0}\right)$, we have

$$
\left[h_{H \epsilon g}^{\operatorname{lin}}(\alpha)\right]=\left[\gamma_{0}\right]
$$

It follows from Lemma 8.3 that

$$
\lambda_{\epsilon g}\left(h_{H \epsilon g}^{l i n}(\alpha)\right) \geq-\max \epsilon g .
$$

From (8.10), we derive

$$
\begin{aligned}
\lambda_{H}(\alpha) & \geq \int_{0}^{1} \min (\epsilon g-H) d t-\max \epsilon g \\
& =\int_{0}^{1}-\max (H-\epsilon g) d t-\max \epsilon g .
\end{aligned}
$$

Letting $\epsilon \rightarrow 0$, we have proved

$$
\lambda_{H}(\alpha) \geq \int_{0}^{1}-\max H d t
$$

and then taking the infimum over $\alpha \in \widetilde{C F}(H)$ with $h_{\epsilon g H}(P D(a))=[\alpha]$, we derive

$$
\rho(H ; a) \geq \int_{0}^{1}-\max H d t
$$


which in particular proves $\rho(H ; a)>-\infty$.

To prove the continuity of $H \mapsto \rho(H ; a)$ in the $C^{0}$-topology, we imitate the above argument by replacing $\epsilon g$ by another generic Hamiltonian $K$. As in (8.10), we have

$$
\lambda_{K}\left(h_{H K}^{l i n}(\alpha)\right) \leq \lambda_{H}(\alpha)+\int_{0}^{1}-\min (K-H) d t .
$$

We have $\left[h_{H K}^{\text {lin }}(\alpha)\right]=\left[h_{\epsilon g K}(P D(a))\right]$ in $H F\left(K ; \Lambda_{\omega}\right)$ because $[\alpha]=h_{\epsilon g H}(P D(a))$ in $H F\left(H ; \Lambda_{\omega}\right)$. From $(8.15)$ and the definition of $\rho(H ; a)$, we have

$$
\rho(K ; a) \leq \lambda_{H}(\alpha)+\int_{0}^{1}-\min (K-H) d t .
$$

This proves

$$
\rho(K ; a) \leq \rho(H ; a)+\int_{0}^{1}-\min (K-H) d t
$$

by taking the infimum of $\lambda_{H}(\alpha)$ over $\alpha$ with $[\alpha]=h_{\epsilon H}(P D(a))$. Equivalently we have

$$
\rho(K ; a)-\rho(H ; a) \leq \int_{0}^{1}-\min (K-H) d t
$$

Next we want to prove

$$
\int_{0}^{1}-\max (K-H) d t \leq \rho(K ; a)-\rho(H ; a) .
$$
get

We apply (8.15) with $H$ and $K$ switched and $\alpha^{\prime}$ with $\left[\alpha^{\prime}\right]=h_{\epsilon g K}(P D(a))$ and

$$
\lambda_{H}\left(h_{K H}^{\operatorname{lin}}\left(\alpha^{\prime}\right)\right) \leq \lambda_{K}\left(\alpha^{\prime}\right)+\int_{0}^{1}-\min (H-K) d t
$$

or

$$
\lambda_{K}\left(\alpha^{\prime}\right)-\lambda_{H}\left(h_{K H}^{l i n}\left(\alpha^{\prime}\right)\right) \geq \int_{0}^{1} \min (H-K) d t=\int_{0}^{1}-\max (K-H) .
$$

Since $\left[\alpha^{\prime}\right]=h_{\epsilon g K}(P D(a))$ and $h_{K H}^{l i n} \circ h_{\epsilon g K}$ is chain homotopic to $h_{\epsilon g H}^{l i n}$, we also have

$$
\left[h_{K H}^{l i n} \alpha^{\prime}\right]=h_{\epsilon g H}(P D(a)) .
$$

Therefore we derive (8.17) from this by the same argument as that of (8.16). Combining (8.16) and (8.17), we have proved

$$
\int_{0}^{1}-\max (K-H) d t \leq \rho(K ; a)-\rho(H ; a) \leq \int_{0}^{1}-\min (K-H) d t .
$$

Now it follows from (8.18) that the function $H \mapsto \rho(H ; a)$ can be extended to $C^{0}(M \times$ $[0,1])$ as a continuous function in $C^{0}$-topology. This finishes the proof.

These numbers $\rho(H ; a)$ will satisfy the properties of the same kind as the invariants constructed by the author in [Oh5]. We refer to $[\mathrm{Oh} 3,5]$ for the statements and proofs of the properties of $\rho$ in the context of Lagrangian submanifolds on the cotangent bundle leaving complete details to [Oh7] for the present case.

We now focus on the special cases where the corresponding class $a$ is the class 1 in $H^{*}(M ; \mathbb{Q})$. 
Definition \& Theorem $8.4[\mathrm{OH} 7]$. Let 1 be the identity class of $H^{*}(M, \mathbb{Q})$. For each given Hamiltonian $H$, we define

$$
\gamma(H)=\rho(H ; 1)+\rho(\bar{H} ; 1) .
$$

Then we have $\gamma(H) \geq 0$, and

$$
\gamma(H)=\gamma(K)
$$

as long as $H \sim K$. This makes $\gamma(H)$ depends only on the equivalence class $[H]$, i.e, defines a well-defined function on the covering space $\pi: \widetilde{\mathcal{H a m}}(M, \omega) \rightarrow \mathcal{H a m}(M, \omega)$. Now for a given Hamiltonian diffeomorphism $\phi$, we define

$$
\gamma(\phi)=\inf _{H \mapsto \phi} \gamma(H)=\inf _{\pi([H])=\phi} \gamma([H])
$$

for any Hamiltonian diffeomorphism $H \mapsto \phi$.

The following theorem has been proven in [Oh7] to which we refer the readers.

TheOREM 8.5 [OH7]. The above function $\gamma: \mathcal{H a m}(M, \omega) \rightarrow \mathbb{R}_{+}$satisfies the following properties:

(1) $\gamma(\phi)=0$ iff $\phi=i d$

(2) $\gamma\left(\phi_{1} \phi_{2}\right) \leq \gamma\left(\phi_{1}\right)+\gamma\left(\phi_{2}\right)$

(3) $\gamma\left(\psi \circ \phi \circ \psi^{-1}\right)=\gamma(\phi)$ for any symplectic diffeomorphism $\psi$.

(4) $\gamma(\phi) \leq\|\phi\|$

This norm reduces to the norm Schwarz constructed in $[\mathrm{Sc}]$ for the symplectically aspherical case where the norm $\gamma$ is defined by

$$
\gamma(H)=\rho(H ; 1)-\rho(H ; \mu)
$$

where $\mu$ is the volume class in $H^{*}(M)$, following $[\mathrm{V}]$ and $[\mathrm{Oh} 5]$. The reason why the two (8.19) and (8.21) coincide is that in the aspherical case, we have the additional identity

$$
\rho(\bar{H}: 1)=-\rho(H ; \mu) .
$$

But Polterovich observed [Po3] that this latter identity fails in the non-exact case due to the quantum contribution. In fact in the non-exact case, even positivity of (8.21) seems to fail. It turns out that our definition (8.19) in Definition 8.4 is the right one to take, which satisfies all the expected properties. We refer readers to [Oh7] for the proof of Theorem 8.5 and for further consequences of the spectral invariants in the study of length minimizing property of Hofer's geodesics and new lower bounds for the Hofer norm of Hamiltonian diffeomorphisms.

Appendix. In this appendix, we prove Proposition 7.13. Since this proposition is a general fact for arbitrary pairs $(G, F)$ of Hamiltonians with $G \sim F$, we gather the facts from the main part of the paper that are needed and make this appendix self-contained.

We first recall the Handle sliding lemma. Let $H$ be any Hamiltonian and consider the Cauchy-Riemann equation

$$
\frac{\partial u}{\partial \tau}+J\left(\frac{\partial u}{\partial t}-X_{H(u)}(u)\right)=0 .
$$


We call a solution $u$ trivial if it is $\tau$-independent, i.e., stationary. We define

$$
A_{(J, H)}:=\inf \left\{\int\left|\frac{\partial u}{\partial \tau}\right|^{2} \mid u \text { satisfies (A.1) and is not trivial }\right\} .
$$

Let $j=\left\{J^{s}\right\}_{0 \leq s \leq 1}$ and the family $\mathcal{H}=\{H(\eta)\}_{\eta \in[0,1]}$ be given. We define

$$
A_{(j, \mathcal{H})}=\inf _{\eta \in[0,1]} A_{\left(J^{\eta}, H(\eta)\right)}
$$

In general, this number could be zero. When it becomes positive, we have the following result. This is an easy version of Proposition 6.3

Proposition A.1. Let $\{H(\eta)\}_{0 \leq \eta \leq 1}$ be a smooth family of Hamiltonians and $j=\left\{J^{s}\right\}$ be a smooth periodic (two parameter) family of compatible almost complex structures. Suppose that $A_{j, \mathcal{H}}$ is positive. Let $\eta_{1}, \eta_{2} \in[0,1]$. Then for any fixed $j$ and for any $\varepsilon>0$, there exists a constant $\delta>0$ such that if $\left|\eta_{1}-\eta_{2}\right|<\delta$, any finite energy solution of

$$
\frac{\partial u}{\partial \tau}+J^{\rho(\tau)}\left(\frac{\partial u}{\partial t}-X_{H^{\rho(\tau)}}(u)\right)=0
$$

must be either satisfies

$$
\int\left|\frac{\partial u}{\partial \tau}\right|^{2} \leq \varepsilon
$$

or

$$
\int\left|\frac{\partial u}{\partial \tau}\right|^{2} \geq A_{(j, \mathcal{H})}-\varepsilon
$$

Here $H^{s}$ is the linear path $H^{s}=(1-s) H\left(\eta_{1}\right)+s H\left(\eta_{2}\right)$ and $\rho$ is the standard function as before.

As in Proposition 6.3, we call a solution $u$ of (A.4) very short if it satisfies (A.4) and long if it satisfies (A.6).

Corollary A.2 [Corollary $6.4, \S 6$ ]. Let $\varepsilon>0$ be any given number. Then there exists $\delta>0$ such that for any $\eta_{1}, \eta_{2}$ with $\left|\eta_{2}-\eta_{1}\right|<\delta$, the following holds: if $u$ is very short, then

$$
\begin{aligned}
-\varepsilon+\int_{0}^{1}-\max _{x}\left(H\left(\eta_{2}\right)-H\left(\eta_{1}\right)\right) d t & \leq \mathcal{A}_{H\left(\eta_{2}\right)}(u(+\infty))-\mathcal{A}_{H\left(\eta_{1}\right)}(u(-\infty)) \\
& \leq \int_{0}^{1}-\min _{x}\left(H\left(\eta_{2}\right)-H\left(\eta_{1}\right)\right) d t
\end{aligned}
$$

If $u$ is not very short, then we have

$$
\mathcal{A}_{H\left(\eta_{2}\right)}(u(+\infty))-\mathcal{A}_{H\left(\eta_{1}\right)}(u(-\infty)) \leq-A_{(j, \mathcal{H})}+\varepsilon+\int_{0}^{1}-\min _{x}\left(H\left(\eta_{2}\right)-H\left(\eta_{1}\right)\right) d t
$$

We would like to apply these results to the path $\mathcal{F}=\left\{F^{s}\right\}_{0 \leq s \leq 1}$. We first prove LEMMA A.3. Let $j=\left\{J^{s}\right\}$ be the family of almost complex structures defined by

$$
J_{t}^{s}=\left(h_{t}^{s}\right)^{*} J_{t} .
$$

Then we have

$$
A_{\left(J^{s}, F^{s}\right)}=A_{(J, G)}
$$


In particular, we have

$$
A_{(j, \mathcal{F})}>0
$$

Proof. We first note that the map

$$
x \mapsto z_{x} ; \quad z_{x}(t):=h_{t}^{s}(x)
$$

and (2.4) give one-one correspondence between $\operatorname{Per}(G)$ and $\operatorname{Per}\left(F^{s}\right)$ and between Crit $\mathcal{A}_{G}$ and Crit $\mathcal{A}_{F^{s}}$ respectively. Furthermore (A.10) also provides one-one correspondence between the solution sets of the corresponding Cauchy-Riemann equations by

$$
u \mapsto u^{s} ; \quad u^{s}(\tau, t)=h_{t}^{s}(u(\tau, t))
$$

And a straightforward calculation shows the identity

$$
\int\left|\frac{\partial u}{\partial \tau}\right|_{J}^{2}=\int\left|\frac{\partial u^{s}}{\partial \tau}\right|_{J^{s}}^{2}
$$

which finishes the proof.

We are now ready to provide the proof of Proposition 7.13. We choose the partition

$$
I: 0=s_{0}<s_{1}<\cdots<s_{N}=1
$$

so that its mesh

$$
\Delta_{I}(\mathcal{F})<\frac{1}{2} \delta_{2}
$$

where $\Delta_{I}(\mathcal{F})$ is defined by

$$
\Delta_{I}(\mathcal{F}):=\inf _{j}\left\{\int_{0}^{1}-\min \left(F^{s_{j+1}}-F^{s_{j}}\right) d t, \int_{0}^{1} \max \left(F^{s_{j+1}}-F^{s_{j}}\right) d t\right\} .
$$

We will prove the proposition in 3 steps: the finiteness, the upper estimates and the lower estimates.

\section{Step 1: the finiteness}

The finiteness of this function follows from the assumption $[\alpha] \neq 0$ and from construction of the chain map. More specifically, the chain map

$$
h_{\mathcal{F}^{s}}^{a d b}: \widetilde{C F}(G) \rightarrow \widetilde{C F}(F)
$$

maps Novikov cycles to Novikov cycles and induces an isomorphism in the homology over the Novikov rings. Since $\left[\alpha_{G}\right] \neq 0$, we have $\left[h_{\mathcal{F}^{s}}^{a d b}\left(\alpha_{G}\right)\right] \neq 0$ and in particular $h_{\mathcal{F}^{s}}^{a d b}\left(\alpha_{G}\right) \neq 0$ for all $s$. Hence comes the finiteness of the level of $h_{\mathcal{F}^{s}}^{a d b}\left(\alpha_{G}\right)$, i.e, the value of $\mu(s)$.

Step 2: the upper estimates

In this step, we will prove

$$
\mu(s)-\mu\left(s^{\prime}\right) \leq \int_{0}^{1}-\min _{x}\left(F^{s}-F^{s^{\prime}}\right) d t
$$

for $s, s^{\prime}$ with $\left|s-s^{\prime}\right| \leq \delta$ for sufficiently small $\delta$. This upper estimates can be proved without help of the Handle sliding lemma. 
We recall that the chain map $h_{\mathcal{F}^{s}}^{a d b}$ is defined as the composition of the chain maps $h_{s_{j+1} s_{j}}^{l i n}: \widetilde{C F}\left(F^{s_{j+1}}\right) \rightarrow \widetilde{C F}\left(F^{s_{j}}\right)$ over the linear homotopy for the partition $I$. We first consider the first segment $\left[0, s_{1}\right]$. In this segment, we have

$$
h_{\mathcal{F} s}^{a d b}=h_{\mathcal{F} s}^{\text {lin }}
$$

over the linear path $u \mapsto(1-u) G+u F^{s}$.

We consider the chain map $h_{s s^{\prime}}^{\mathcal{F}, \text { lin }}$ which is induced by the assignment

$$
h_{s s^{\prime}}^{\mathcal{F}, l i n}\left(\left[z^{-}, w^{-}\right]\right)=\sum_{\left[z^{+}, w^{+}\right] \in \operatorname{Crit} \mathcal{A}_{F^{s^{\prime}}}} \#\left(\mathcal{M}_{J}^{\mathcal{G}_{1}}\left(\left[z^{-}, w^{-}\right],\left[z^{+}, w^{+}\right]\right)\right)\left[z^{+}, w^{+}\right]
$$

for each $\left[z^{-}, w^{-}\right] \in \operatorname{Crit} \mathcal{A}_{F^{s}}$. Here $\mathcal{M}_{J}^{\mathcal{G}_{1}}\left(\left[z^{-}, w^{-}\right],\left[z^{+}, w^{+}\right]\right)$denotes the moduli space of trajectories of the Cauchy-Riemann equation

$$
\frac{\partial u}{\partial \tau}+J^{\rho(\tau)}\left(\frac{\partial u}{\partial t}-X_{F^{\rho(\tau)}}(u)\right)
$$

and $\#\left(\mathcal{M}_{J}^{\mathcal{G}_{1}}\left(\left[z^{-}, w^{-}\right],\left[z^{+}, w^{+}\right]\right)\right)$denotes its (rational) Euler number (see [FOn], [LT], $[\mathrm{Ru}]$ for the precise meaning). In the case relevant to the chain map the moduli space is zero-dimensional. In particular, if this number is not zero, then (A.13) has a solution.

Assuming the existence of such pair $\left[z^{-}, w^{-}\right] \in \operatorname{Crit} \mathcal{A}_{F^{s}}$ and $\left[z^{+}, w^{+}\right] \in \operatorname{Crit} \mathcal{A}_{F^{s^{\prime}}}$ for the moment, we proceed with the proof. Then to every pair $\left[z^{-}, w^{-}\right]$and $\left[z^{+}, w^{+}\right]$ for which $\#\left(\mathcal{M}_{J}^{\mathcal{G}_{1}}\left(\left[z^{-}, w^{-}\right],\left[z^{+}, w^{+}\right]\right)\right)$is non-zero, we have

$$
\mathcal{A}_{F^{s^{\prime}}}(u(\infty))-\mathcal{A}_{F^{s}}(u(-\infty)) \leq \int_{0}^{1}-\min _{x}\left(F^{s^{\prime}}-F^{s}\right) d t
$$

Taking the maximum over $\left[z^{-}, w^{-}\right]$among the generators of $h_{0 s}^{\mathcal{F}, l i n}\left(\alpha_{G}\right)$, we get

$$
\mathcal{A}_{\eta^{\prime} g}(u(\infty))-\mu_{1}(\eta) \leq \int_{0}^{1}-\min _{x}\left(F^{s^{\prime}}-F^{s}\right) d t .
$$

Since this holds for any generator $\left[z^{+}, w^{+}\right]=u(\infty)$ of $h_{0 s^{\prime}}^{\mathcal{F}, \text { lin }}\left(\alpha_{G}\right)$, (A.15)) proves (A.12) by the definition of $\mu$.

Now it remains to prove the existence of a pair, $\left[z^{-}, w^{-}\right] \in \operatorname{Crit} \mathcal{A}_{F^{s}}$ and $\left[z^{+}, w^{+}\right] \in$ Crit $\mathcal{A}_{F^{s^{\prime}}}$, such that

$$
\#\left(\mathcal{M}_{J}^{\mathcal{G}_{1}}\left(\left[z^{-}, w^{-}\right],\left[z^{+}, w^{+}\right]\right)\right) \neq 0
$$

and $\left[z^{-}, w^{-}\right]$contributes $h_{0 s}^{\mathcal{F}, l i n}\left(\alpha_{G}\right)$ and $\left[z^{+}, w^{+}\right]$contributes $h_{0 s}^{\mathcal{F}, l i n}\left(\alpha_{G}\right)$. We recall that

$$
h_{0 s^{\prime}}^{\mathcal{F}, \text { lin }}-h_{s s^{\prime}}^{\mathcal{F}, l i n} \circ h_{0 s}^{\mathcal{F}, l i n}=\partial_{F^{s^{\prime}}} \circ \widetilde{H}+\widetilde{H} \circ \partial_{F^{s}}
$$

where $\widetilde{H}$ is defined by considering parameterized equation induced by the homotopy (of homotopies) $\overline{\mathcal{L}}=\left\{\mathcal{L}_{\kappa}\right\}_{\kappa}$ connecting the linear homotopy between $F^{0}=G$ and $F^{s}$ and the glued homotopy via $0 \mapsto s \mapsto s^{\prime}$. However if $s$ is close to $s^{\prime}$ and the CauchyRiemann equation for $\mathcal{L}_{0}$ is regular, then those corresponding to $\mathcal{L}_{k}$ are all regular for $0 \leq \kappa \leq 1$. Since $\widetilde{H}$ is defined by counting generic non-regular solutions on $\kappa \in(0,1)$, this proves that $\widetilde{H}=0$ if $s$ is very close to $s^{\prime}$. Therefore we have

$$
h_{0 s^{\prime}}^{\mathcal{F}, \text { lin }}\left(\alpha_{G}\right)=h_{s s^{\prime}}^{\mathcal{F}, \text { lin }} \circ h_{0 s}^{\mathcal{F}, \text { lin }}\left(\alpha_{G}\right)
$$


if $\left|s-s^{\prime}\right|<\delta$ for sufficiently small $\delta$. By the definition of the chain map $h_{s s^{\prime}}^{\mathcal{F}, \text { in }}$, there must be such a pair of $\left[z^{-}, w^{-}\right]$and $\left[z^{+}, w^{+}\right]$for which (A.15) holds. This finishes the proof of (A.12).

Step 3: the lower estimate

This is the place where the Handle sliding lemma plays a crucial role. We apply $h_{s^{\prime} s}^{\mathcal{F}^{-1}, \text { lin }}$ to (A.17) to get

$$
h_{s^{\prime} 0}^{\mathcal{F}^{-1}, \text { lin }} \circ h_{0 s^{\prime}}^{\mathcal{F}, l i n}\left(\alpha_{G}\right)=h_{s^{\prime} 0}^{\mathcal{F}^{-1}, l i n} \circ h_{s s^{\prime}}^{\mathcal{F}, l i n} \circ h_{0 s}^{\mathcal{F}, l i n}\left(\alpha_{G}\right) .
$$

Therefore $h_{s^{\prime} 0}^{\mathcal{F}^{-1}, \text { lin }} \circ h_{s s^{\prime}}^{\mathcal{F}, l i n} \circ h_{0 s}^{\mathcal{F}, l i n}\left(\alpha_{G}\right)$ is homologous to $\alpha_{G}$ in $\widetilde{C F}(G)$ because $h_{s^{\prime} 0}^{\mathcal{F}^{-1}, \text { lin }} \circ$ $h_{0 s^{\prime}}^{\mathcal{F}, l i n}\left(\alpha_{G}\right)$ is so. By the Non pushing-down lemma, Proposition 7.14, we have

$$
\lambda_{\epsilon_{0} G^{\epsilon_{0}}}\left(h_{s^{\prime} 0}^{\mathcal{F}^{-1}, \text { lin }} \circ h_{s s^{\prime}}^{\mathcal{F}, l i n} \circ h_{0 s}^{\mathcal{F}, l i n}\left(\alpha_{G}\right)\right) \geq \lambda_{G}\left(\alpha_{G}\right)=c^{+} .
$$

This gives rise to

$$
\begin{aligned}
\lambda_{F^{s^{\prime}}}\left(h_{s s^{\prime}}^{\mathcal{F}, l i n} \circ h_{0 s}^{\mathcal{F}, l i n}\left(\alpha_{G}\right)\right) & \geq \lambda_{G}\left(\alpha_{G}\right)-\frac{1}{2} \delta_{2} \\
& \geq \lambda_{F^{s}}\left(h_{0 s}^{\mathcal{F}, l i n}\left(\alpha_{G}\right)\right)-\delta_{2} .
\end{aligned}
$$

Now we choose $\delta_{2}$ so small in (7.4) that we have

$$
\delta_{2}<\min \left\{\frac{1}{2} A_{(j, \mathcal{F})}, \frac{1}{2} A_{\left(j^{-1}, \mathcal{F}^{-1}\right)}\right\} .
$$

Then the trajectory constructed in Step 2 that satisfies (A.15) must be very short. On the other hand for the very short trajectories, the lower estimate (A.7) holds.

Combining Step 1-3, we have proved that the function $\mu$ is continuous and so must be constant on $\left[0, s_{1}\right]$. Then this also implies Non pushing-down lemma for $F^{s_{1}}$ from which we can repeat the above argument to the segment $\left[s_{1}, s_{2}\right]$. We repeat this to all $j=3, \cdots, N-1$ which finishes the proof of Proposition 7.13.

\section{REFERENCES}

[B] BANYAGA, A., Sur la structure du groupe des difféomorphismes qui préservent une forme symplectique, Comm. Math. Helv., 53 (1978), pp. 174-227.

[BP] Bialy, M. And Polterovich, L., Geodesics of Hofer's metric on the group of Hamiltonian diffeomorphisms, Duke J. Math., 76 (1994), pp. 273-292.

[Ch] Chekanov, Y., Lagrangian intersections, symplectic energy and areas of holomorphic curves, Duke J. Math., 95 (1998), pp. 213-226.

[En] Entov, M., K-area, Hofer metric and geometry of conjugacy classes in Lie groups, Invent. Math., 146 (2001), pp. 93-141.

[Fl1] Floer, A., Witten's complex and infinite dimensional Morse theory, J. Differ. Geom., 30 (1989), pp. 207-221.

[Fl2] Floer, A., Symplectic fixed point and holomorphic sphere, Commun. Math. Phys., 120 (1989), pp. $575-611$.

[FHS] Floer, A., Hofer, H. ANd Salamon, D., Transversality in elliptic Morse theory for the symplectic action, Duke Math. J., 80 (1995), pp. 251-292.

[FOh] FUKAYA, K. AND OH, Y.-G., Zero-loop open strings in the cotangent bundle and Morse homotopy, Asian J. Math., 1 (1997), pp. 99-180.

[FOOO] FUKAYA, K., OH, Y.-G., OHTA, H. AND ONO, K., Lagrangian intersection Floer theory anomaly and obstruction-, preprint, Kyoto University, (2000). 
[FOn] Fukaya, K. And Ono, K., Arnold conjecture and Gromov-Witten invariants, Topology, 38 (1999), pp. 933-1048.

[H1] HOFER, H., On the topological properties of symplectic maps, Proc. Royal Soc. Edinburgh, 115 (1990), pp. 25-38.

[H2] Hofer, H., Estimates for the energy of a symplectic map, Comment. Math. Helv., 68 (1993), pp. 48-72.

[HS] Hofer, H., SAlamon, D., Floer homology and Novikov rings, in Floer Memorial Volume, Hofer, H. et al, eds., Birkhäuser, 1995, pp. 483-524.

[Lee] LEE, Y. J., Reidemeister torsion in symplectic Floer theory and counting pseudo-holomorphic tori, preprint, math.DG/0111313.

[LM1] Lalonde, F. AND McDuff, D., The geometry of symplectic energy, Annals of Math., 141 (1995), pp. 349-471.

[LM2] Lalonde, F. AND MCDuff, D., Hofer's $L^{\infty}$-geometry: energy and stability of Hamiltonian flows I, II, Invent. Math. (1995), pp. 1-33, 35-69.

[LT] Liu, G. ANd Tian, G., Floer homology and Arnold conjecture, J. Differ. Geom., 49 (1998), pp. 1-74.

[Mc] McDuff, D., Geometric variants of the Hofer norm, preprint, 2001.

[MO1] Milinković, D. AND OH, Y.-G., Floer homology and the stable Morse homology, J. Korean Math. Soc., 34 (1997), pp. 1065-1087.

[MO2] Milinković, D. AND OH, Y.-G., Generating functions versus action functional, in CRM Proceedings and Lecture Notes 15, AMS, 1998, pp. 107-125.

[MS] MCDuff, D. AND Slimowitz, J., Hofer-Zehnder capacity and length minimizing Hamiltonian paths, preprint, 2000.

[Oh1] Он, Y.-G., Floer cohomology, spectral sequences, and the Maslov class of Lagrangian embeddings, Internat. Math. Res. Notes, No. 7 (1996), pp. 305-346.

[Oh2] OH, Y.-G., Relative Floer and quantum cohomology and the symplectic topology of Lagrangian submanifolds, in Contact and Symplectic Geometry, C. Thomas, ed., Cambridge Univ. Press., Cambridge UK, 1996, p. $201-267$.

[Oh3] Он, Y.-G., Symplectic topology as the geometry of action functional, I, Jour. Differ. Geom., 46 (1997), pp. 499-577.

[Oh4] OH, Y.-G., Gromov-Floer theory and disjunction energy of compact Lagrangian embeddings, Math. Rec. Lett., 4 (1997), pp. 895-905.

[Oh5] OH, Y.-G., Symplectic topology as the geometry of action functional, II, Commun. Anal. Geom., 7 (1999), pp. 1-55.

[Oh6] Он, Y.-G., Normalization of the Hamiltonian and the action spectrum, preprint, April 2002, math.SG/0206090.

[Oh7] Он, Y.-G., Mini-max theory, spectral invariants and geometry of the Hamiltonian diffeomorphism group, preprint, June 2002, math.SG/0206092.

[Po1] Polterovich, L., Symplectic displacement energy for Lagrangian submanifolds, Ergodic Theory and Dynamical Systems, 13 (1993), pp. 357-367.

[Po2] Polterovich, L., The geometry of the group of symplectic diffeomorphisms (to appear), Birkhäuser.

[Po3] Polterovich, L., private communication.

[Ru] RUAN Y., Virtual neighborhood and pseudo-holomorphic discs, Turkish J. Math., 23 (1999), pp. 161-231.

[Sc] SCHWARz, M., On the action spectrum for closed symplectically aspherical manifolds, Pacific J. Math., 193 (2000), pp. 419-461.

[Se] SEIDEL, P., $\pi_{1}$ of symplectic diffeomorphism groups and invertibles in quantum homology rings, GAFA (1997), pp. 1046-1095.

[Si] SiBURG, K., New minimal geodesics in the group of symplectic diffeomorphisms, Calc. Var., 3 (1995), pp. 299-309.

[V] Viterbo, C., Symplectic topology as the geometry of generating functions, Math. Ann., 292 (1992), pp. 685-710. 